\title{
Penalidades Exatas para Desigualdades Variacionais
}

Thiago Afonso de André

\author{
DISSERTAÇÃO APRESENTADA \\ $\mathrm{AO}$ \\ INSTITUTO DE MATEMÁTICA E ESTATÍSTICA \\ DA \\ UNIVERSIDADE DE SÃO PAULO \\ PARA \\ OBTENÇÃO DO TÍTULO DE MESTRE \\ $\mathrm{EM}$ \\ CIÊNCIAS
}

Área de Concentração: Matemática Aplicada

Orientador: Prof. Dr. Paulo José da Silva e Silva

Durante a elaboração deste trabalho o autor recebeu auxílio financeiro da FAPESP

São Paulo, fevereiro de 2007 


\title{
Penalidades Exatas para Desigualdades Variacionais
}

\author{
Este exemplar corresponde à redação final \\ da dissertação devidamente corrigida e \\ defendida por Thiago Afonso de André e \\ aprovada pela comissão julgadora.
}

São Paulo, fevereiro de 2007.

Banca examinadora:

Prof. Dr. Paulo José da Silva e Silva

IME-USP

Prof. Dr. Marcelo Gomes de Queiroz

IME-USP

Prof. Dr. Roberto Andreani

IMECC-UNICAMP 


\section{Agradecimentos}

A minha mãe e minha irmã, por tudo, mas principalmente pelo eterno apoio.

Ao meu pai, pela inspiração e pelas memórias.

Ao Paulo, pela sua incrível paciência, perseverança, apoio, dedicação e genialidade.

A Cidinha, Josenilton, Mané, Sônia e Barone, meus grandes professores, por todas as lições e por todo incentivo.

A Lu, por toda força e suporte nos momentos finais.

Aos meus amigos, pelas risadas, provocações e abraços. 


\section{Resumo}

Esta dissertação busca aproveitar os métodos de penalidades exatas diferenciáveis de programação não-linear para resolver problemas de desigualdades variacionais. Problemas desse tipo têm recebido grande atenção na literatura recentemente e possuem aplicações em diversas áreas como Engenharia, Física e Economia [21].

Métodos de penalidades exatas diferenciáveis foram desenvolvidos nos anos 70 e 80 para resolver problemas de otimização com restrições por meio da solução de problemas irrestritos. Esses problemas são tais que, com uma escolha apropriada do parâmetro de penalização, uma solução do problema original é recuperada após a resolução de um único problema irrestrito. A função a ser minimizada é semelhante a um lagrangiano aumentado clássico, porém uma estimativa do multiplicador é automaticamente calculada a partir do ponto primal.

Nesse trabalho, mostramos como acoplar a estimativa de multiplicadores sugeridas por Glad e Polak [27] ao lagrangiano aumentado clássico para desigualdades variacionais sugerido por Auslender e Teboulle [2]. Obtivemos assim uma penalidade exata para problemas de desigualdades variacionais. Os resultados mais finos de exatidão foram obtidos no caso de problemas de complementaridade não-linear. Uma característica importante da penalidade proposta é que ela não envolve informações de segunda ordem das funções que definem a desigualdade variacional.

Além desses resultados, que formam o núcleo da dissertação, apresentamos uma breve revisão de penalidades não-exatas diferenciáveis , exatas não-diferenciáveis e exatas diferenciáveis em otimização. 


\begin{abstract}
This work intends to build upon differentiable exact penalty methods for nonlinear programming, using them to solve variational inequality problems. Such problems have been given a lot of attention in the literature lately and have applications to diverse areas of knowledge such as Engineering, Physics and Economics.

Differentiable exact penalty methods were developed during the 70s and 80s to solve constrained optimization problems by means of the solution of unconstrained problems. Those problems are such that, with an appropriate choice of the penalty parameter, one finds a solution of the original constrained problem by solving only one unconstrained problem. The function which is minimized is similar to the classic augmented lagrangian, but an estimate of the multiplier is automatically calculated from the primal point.

In this thesis we show how to couple Glad and Polak's multiplier estimate, [27], with the classic augmented lagrangian of a variational inequality developed by Auslender and Teboulle [2]. This allowed us to obtain an exact penalty function for variational inequality problems. The best exactness results were obtained in the particular case of nonlinear complementarity problems. An important characteristic of the proposed penalty is that it doesn't involve second order information of any of the functions which compose the variational inequality.

In addition to those results, which are the core of this work, we also present a brief review of inexact differentiable penalties, exact nondifferentiable penalties and differentiable exact penalties in optimization.
\end{abstract}




\section{Sumário}

1 Introdução $\quad 3$

2 Penalidades Exatas para Otimização $\quad 8$

2.1 Penalidades "Não-Exatas" Diferenciáveis . . . . . . . . . . . . . . . . . 8

2.2 Penalidades Exatas Não-Diferenciáveis ～. . . . . . . . . . . . . . . . . 10

$2.2 .1 \quad$ Dualidade Lagrangiana $\ldots \ldots \ldots \ldots \ldots$

2.2 .2 Sensibilidade e a função primal . . . . . . . . . . . . . . . . . . . 13

2.2.3 Penalidade Exata Não-Diferenciável . . . . . . . . . . . . . . . . . . 14

2.2 .4 Definições de Exatidão . . . . . . . . . . . . . . . . . . . . . 17

2.3 Primeiras Penalidades Exatas Diferenciáveis: Restrições de Igualdade . . . . 19

2.3.1 Eliminando os multiplicadores . . . . . . . . . . . . . . . 22

2.3.2 A relação com métodos de multiplicadores . . . . . . . . . . . . . . . . 24

2.4 Penalidades Exatas para Desigualdades . . . . . . . . . . . . . . 24

2.4 .1 Uma penalidade especial . . . . . . . . . . . . . . . . 27

3 Penalidades Exatas para Desigualdades Variacionais $\quad 37$

3.1 Desigualdades Variacionais . . . . . . . . . . . . . . . . . 37

3.1.1 Métodos para resolver Desigualdades Variacionais _. . . . . . . . 38

3.2 Estendendo Penalidades Exatas para Desigualdades Variacionais . . . . . . . . 42

3.2.1 O Lagrangiano Aumentado de Desigualdades Variacionais . . . . . . . 44 
3.2.2 O sistema KKT de Desigualdades Variacionais . . . . . . . . . . . . . 44

3.2.3 Exatidão da Penalidade . . . . . . . . . . . . . . . . . . 46

3.3 Um Caso Especial: Complementaridade Não-Linear . . . . . . . . . . . . . . 50

4 Conclusão

A Métodos Numéricos

A.1 Reformulações NCP . . . . . . . . . . . . . . . . . . . 57

A.2 Métodos de Resolução . . . . . . . . . . . . . . . . . . . . . . . . . . . . . 59

A.3 Resultados numéricos . . . . . . . . . . . . . . . . . . . 60

A.3.1 Melhorando a Robustez . . . . . . . . . . . . . . . . . . . 61

$\begin{array}{ll}\text { Referências Bibliográficas } & 64\end{array}$ 


\section{Capítulo 1}

\section{Introdução}

Modelos de otimização têm sido bastante utilizados na análise e solução de problemas vindos das mais diversas áreas do conhecimento como Física, Engenharia, Economia ou Química. Seu estudo e o desenvolvimento de algoritmos para sua solução é, portanto, uma área importante da Matemática que tem visto um rápido avanço nas últimas décadas. Por outro lado, uma generalização de otimização que foca nas relações equilíbrio descritas pelas condições de otimalidade de primeira ordem, conhecidas como desigualdades variacionais, têm mais recentemente recebido uma grande atenção da comunidade de pesquisa.

Este trabalho visa explorar a relação entre otimização e desigualdades variacionais com o objetivo de transpor algoritmos para solução de problemas de programação matemática para um contexto mais geral. Em particular, focamos na extensão de métodos de otimização baseados em penalidades exatas diferenciáveis.

Considere o seguinte problema,

$$
\begin{array}{r}
\min f(x) \\
\text { s.a } g(x) \leq 0 \\
h(x)=0,
\end{array}
$$

em que $x$ é um vetor do $\mathbb{R}^{n}$ e $f: \mathbb{R}^{n} \rightarrow \mathbb{R}, g: \mathbb{R}^{n} \rightarrow \mathbb{R}^{m}$ e $h: \mathbb{R}^{n} \rightarrow \mathbb{R}^{p}$ são funções continuamente diferenciáveis. Esse será o nosso modelo de problema de programação nãolinear com restrições.

É muito comum resolver tais problemas com métodos baseados em penalidades. Estes substituem um problema com restrições por uma seqüência de minimizações sem restrições, 
cujas soluções convergem para uma solução do (NLP). Para resolver problemas irrestritos, como os que aparecem a cada etapa das estratégias baseadas em penalidades, já se conhece uma série de métodos com boas propriedades de convergência [33]. Isso faz com que muitas vezes seja mais eficaz resolver essa seqüência de problemas mais simples do que tentar atacar diretamente o problema (NLP).

Uma das variantes do método de penalidades mais usadas são os métodos dos multiplicadores que surgem a partir da aplicação de métodos de ponto proximal ao dual lagrangiano do problema $[8,40]$.

Outra opção é o uso de penalidades exatas, construídas de modo que todo o problema (NLP) seja transformado em um único problema irrestrito. Por exemplo, pode-se verificar que se $f, g$ e $h$ obedecem certas condições, a resolução de

$$
\min _{x \in \mathbb{R}^{n}} \phi(x, \mu) \stackrel{\text { def }}{=} f(x)+\mu \max \left\{0, g_{1}(x), g_{2}(x), \ldots, g_{m}(x),\left|h_{1}(x)\right|,\left|h_{2}(x)\right|, \ldots,\left|h_{p}(x)\right|\right\}
$$

recupera uma solução de (NLP), contanto que o parâmetro $\mu$ seja suficientemente grande [9]. Infelizmente, essa penalidade exata herda a não-diferenciabilidade da função máximo, o que obriga a utilização de métodos especiais na sua minimização. Ainda, determinar o valor de $\mu$ para que haja essa equivalência de soluções também não é tarefa fácil, como veremos adiante.

Para contornar a não-diferenciabilidade da função acima, muitas outras propostas de penalidades exatas foram feitas, mas dessa vez valendo-se de formulações diferenciáveis. A história do desenvolvimento das penalidades exatas diferenciáveis começa com Fletcher, em 1970, quando ele publica uma série de três artigos que buscavam automatizar a escolha do multiplicador no método de lagrangianos aumentados. Estes trabalhos lidavam apenas com restrições de igualdade $[23,26,24]$. Fletcher criou uma forma de estimar bons multiplicadores a partir de um ponto primal, definindo uma função $\lambda(x)$ e transformando o método seqüencial em um único problema: a minimização do lagrangiano aumentado associado ao problema original usando tais estimativas como multiplicadores

$$
\min _{x \in \mathbb{R}^{n}} f(x)+\lambda(x)^{T} h(x)+c_{k}\|h(x)\|^{2} .
$$

Apesar da análise de convergência e dos testes numéricos publicados, o método não foi amplamente aceito e utilizado pois não havia uma forma fácil de estimar o parâmetro $c_{k} \mathrm{e}$ cada avaliação da função de cálculo do multiplicador era complexa e demorada. Mukai e Polak, em 1975, criaram uma fórmula explícita para calcular $\lambda(x)$ e um algoritmo com critérios para 
controlar o aumento do parâmetro $c_{k}$, além de provar a existência de um limite finito $\bar{c}$, que, uma vez atingido por $c_{k}$, faria com que o lagrangiano aumentado recuperasse as soluções do problema original [31].

Em 1979, Di Pillo e Grippo publicaram uma nova formulação para a penalidade que simplifica muito a análise dos métodos e os aproxima dos métodos de multiplicadores [14]. Isso é possível, pois eles se baseiam em uma função que simplesmente acrescentava ao lagrangiano aumentado um novo termo, penalizando a violação das condições de primeira ordem,

$$
w\left(x, \lambda ; c_{k}\right)=f(x)+\lambda^{T} h(x)+c_{k}\|h(x)\|^{2}+\|M(x)(\nabla f(x)+\nabla h(x) \lambda)\|^{2} .
$$

Essa função deve ser então minimizada irrestritamente com relação a $x$ e $\lambda$. Tecnicamente, ela não é uma penalidade, mas sim um lagrangiano aumentado exato, pois depende explicitamente dos valores de $\lambda$ no espaço dual dos multiplicadores. Mas uma escolha apropriada da matriz $M(\cdot)$ faz com que a função acima fique quadrática em $\lambda$. Nesse caso é possível isolar o multiplicador, ou seja, ao minimizar $w\left(\cdot ; c_{k}\right)$ podemos minimizar inicialmente a função em $\lambda$ e escrever tal solução como uma função de $x$. Assim recuperamos a penalidade de Fletcher e os resultados de Mukai e Polak.

Outra vantagem da formulação de Di Pillo e Grippo é que ela pode também tratar as restrições de desigualdade através da introdução de variáveis auxiliares, usando a transformação clássica $h_{i}(x)=g_{i}(x)+z_{i}^{2}$. Uma nova escolha apropriada de $M(\cdot)$ permite deixar o problema biquadrático em $z$ e assim separar sua minimização, recuperando um problema só em $x$ e $\lambda$. Mas nesse caso não é mais possível isolar $\lambda$ como função de $x$.

Por outro lado, Fletcher adapta sua idéia para desigualdades ainda em 1973. Porém, o problema não possui boas propriedades de diferenciabilidade [25]. Em 1979, Glad e Polak publicam um método de multiplicadores para desigualdades que mostra como controlar o crescimento do parâmetro $c_{k}[27]$.

Finalmente, em 1985 e 1989, novamente Di Pillo e Grippo sistematizam a formulação de Glad e Polak e criam para o caso de desigualdades uma penalidade exata diferenciável que só depende das variáveis primais do problema $[15,16]$.

Esses dois artigos são o ponto de partida de nosso trabalho. Tentaremos aqui estender as idéias de Di Pillo e Grippo para as desigualdades variacionais com restrições funcionais. Nesse contexto, só nos interessa tratar de restrições de desigualdade. Os resultados obtidos também podem ser trazidos de volta ao contexto de programação não-linear.

É importante notar que apesar de tratarmos de penalidades exatas, que teoricamente 
encontram uma solução do problema original em uma minimização, ainda há um parâmetro $c_{k}$ a ser ajustado. Não há uma forma fácil de se determinar o limiar mínimo de $c_{k}$ para que haja a equivalência das soluções do problema original e de sua penalidade exata. Ainda, um aumento exagerado do parâmetro pode tornar a solução numérica do problema desnecessariamente mais trabalhosa. Nos algoritmos comumente encontrados na literatura, a estratégia adotada para se obter uma solução envolve então um processo seqüencial com possível atualização de $c_{k}$ a cada passo $[27,31]$.

Para introduzir o problema de desigualdade variacional, considere $x \in \mathcal{F}$ que satisfaz as condições geométricas de otimalidade associadas ao problema de programação não-linear convexo

$$
\forall y \in \mathcal{F}, \nabla f(x)^{T}(y-x) \geq 0
$$

em que $\mathcal{F}$ representa o conjunto de soluções viáveis. O problema de desigualdade variacional pode ser visto como uma generalização da desigualdade acima em que o gradiente da função objetivo é substituído por uma função $F: \mathbb{R}^{n} \rightarrow \mathbb{R}^{n}$ contínua qualquer. Isto é, dado $\mathcal{F} \subset \mathbb{R}^{n}$ convexo, queremos encontrar $x \in \mathcal{F}$ tal que

$$
\forall y \in \mathcal{F}, F(x)^{T}(y-x) \geq 0
$$

Grande parte dos algoritmos para a solução de problemas de programação não-linear, são capazes apenas de encontrar pontos estacionários, ou seja, pontos que obedecem a condições de primeira ordem. Dessa forma, é natural buscar generalizações de tais métodos para resolver o problema de desigualdade variacional. Entretanto, nesse contexto mais amplo perde-se a função de mérito natural dada pela função objetivo. Os algoritmos devem então ser adaptados.

Nesse sentido, houve um grande esforço de pesquisa para trazer ao contexto de desigualdades variacionais conceitos típicos de otimização. No nosso trabalho, uma idéia importante é a noção de dualidade. Ainda em 1972, Mosco apresenta a primeira versão de um problema dual associado a equações generalizadas, em particular desigualdades variacionais [30]. Esses resultados são estendidos por Attouch e Théra em 1996 [1] e depois trazidos para um contexto bastante genérico por Pennanen em sua tese de doutorado [34, 35].

Recentemente, Eckstein e Ferris aproveitaram tais desenvolvimentos teóricos para estender as idéias de lagrangianos aumentados e o método de multiplicadores para problemas de complementaridade mistos, que são casos particulares de desigualdades variacionais [18]. Em seguida, Auslender e Teboulle estendem ainda mais tais resultados para lidar com restrições descritas por desigualdades convexas gerais [2]. 
Esses trabalhos são o ponto de partida dessa dissertação. Nela buscamos explorar a conexão entre lagrangianos aumentados e penalidades exatas para otimização e a existência de lagrangianos aumentados para desigualdades variacionais com o objetivo de obter penalidades exatas para o problema mais geral.

O restante dessa dissertação é organizado da seguinte maneira. No capítulo 2 apresentamos uma revisão dos resultados de penalidades exatas para otimização e sua relação com lagrangianos aumentados. O capítulo 3 é o núcleo da dissertação. É nele em que apresentamos a extensão de penalidades exatas para problemas de desigualdades variacional e provamos alguns resultados de exatidão das penalidades propostas. Cabe ressaltar que esses resultados são originais.

Ao final adicionamos um apêndice com os resultados de testes numéricos preliminares para o problema de complementaridade não-linear. 


\section{Capítulo 2}

\section{Penalidades Exatas para Otimização}

Este capítulo apresenta uma revisão dos conceitos de penalidade para a solução de problemas de otimização, com enfoque em penalidades exatas diferenciáveis. Ele está organizado da seguinte maneira: a seção 2.1 apresenta a idéia de penalidades para problemas de programação não-linear destacando algumas de suas limitações, a seção 2.2 lida com penalidades exatas não-diferenciáveis. A seção 2.3 apresenta as primeiras penalidades exatas diferenciáveis, com ênfase em problemas com restrições de igualdade. Já a última seção estende tais conceitos para problemas com restrições de desigualdade.

\subsection{Penalidades "Não-Exatas" Diferenciáveis}

Entre os métodos bastante usados para resolver problemas de programação não-linear com restrições estão os métodos clássicos de penalidades como, por exemplo, o de penalidades quadráticas[9], que resolve problemas na forma

$$
\min P_{q}(x ; \alpha, \beta)=f(x)+\alpha\|h(x)\|^{2}+\beta\left\|(g(x))_{+}\right\|^{2} .
$$

Aqui $\gamma_{+}=\max \{\gamma, 0\}$ coordenada a coordenada, e $\alpha$ e $\beta$ são parâmetros estritamente positivos atualizados a cada passo, que visam trazer a solução para o conjunto viável. Uma solução do problema acima não precisa necessariamente satisfazer $g(x) \leq 0$ ou $h(x)=0$, mas ao aumentarmos $\alpha$ e $\beta$, fica cada vez mais vantajoso que essas condições sejam satisfeitas, ou mais custoso que elas sejam violadas. Esse é um exemplo de penalidade externa, em que as 
soluções são possivelmente inviáveis. Quando as restrições são somente de desigualdade, é possível também empregar penalidades internas, que são definidas apenas no conjunto viável. Um exemplo é a penalidade logarítmica $P_{l o g}(x ; \gamma)=f(x)-\frac{1}{\gamma} \log (-g(x))$ [9]. Em ambos os casos, modificar os parâmetros de penalização pode tornar a solução mais próxima da solução do problema original, mas ao custo de tornar o sub-problema numericamente mais difícil de resolver [8].

É importante também notar que dependendo de $f, g$ e $h$, não há nenhum valor de $\alpha, \beta$ ou $\gamma$ que recupere a solução do problema original.

Exemplo 2.1. Seja $f(x)=x_{1}$, com $x \in \mathbb{R}^{2}$. Sejam $g(x)=-x$ e $h(x)=x_{1}-x_{2}$, ou seja, $x \geq 0$ e $x_{1}=x_{2}$ são as restrições e o mínimo é claramente encontrado na origem e tem valor $f(0,0)=0$.

A penalidade quadrática fica

$$
P_{q}(x ; \alpha, \beta)=x_{1}+\alpha\left\|x_{1}-x_{2}\right\|^{2}+\beta\left\|(-x)_{+}\right\|^{2} .
$$

Notando que $\left\|(g(x))_{+}\right\|^{2}$ é diferenciável, sempre que $g$ for, segue então a diferenciabilidade de $P_{q}$. Nesse caso, $\frac{\partial}{\partial x_{1}} P_{q}(0,0 ; \alpha, \beta)=1$ e $\frac{\partial}{\partial x_{2}} P_{q}(0,0 ; \alpha, \beta)=0$, temos que na direção $(-1,0)$, $P_{q}$ é estritamente decrescente. Como $P_{q}(0,0 ; \alpha, \beta)=0$, para todo $\alpha, \beta>0$, vemos que $P_{q}$ atinge valores negativos em pontos arbitrariamente próximos da origem, que portanto nunca é mínimo da penalidade, nem mesmo localmente.

Já a penalidade logarítmica, se nos restringirmos ao caso em $\mathbb{R}, f(x)=x$ e $g(x)=-x$ fica

$$
P_{l o g}(x ; \gamma)=x-\frac{1}{\gamma} \log (x)
$$

Podemos calcular facilmente o mínimo dessa função estritamente convexa igualando sua derivada a zero: $1-\frac{1}{\gamma} \frac{1}{x}=0$ e portanto o minimo é encontrado em $\bar{x}=\frac{1}{\gamma}$, estritamente positivo. Mais uma vez para todo valor do parâmetro a solução em $\bar{x}=0$ nunca é atingida.

Mesmo em um caso só com funções lineares, e portanto diferenciáveis e convexas, já notamos que ao usar os métodos de penalidades tradicionais será necessário levar os parâmetros de penalização a infinito e gerar uma seqüência de soluções da penalidade para cada parâmetro que convergirá, espera-se, para a solução do problema original.

Está claro então o interesse pelo desenvolvimento de estratégias que, com parâmetros finitos, recuperem soluções exatas do problema original. Tais métodos serão chamados de 
métodos de penalidades exatas, em contraposição aos seqüenciais que aqui vimos.

Para terminar, vejamos que mesmo essa convergência da seqüência infinita de soluções não está garantida, principalmente na ausência de convexidade, como mostram os exemplos a seguir.

Exemplo 2.2. Seja $f(x)=\frac{1}{3} x^{3}$, com $x \in \mathbb{R}$. Sem perda de generalidade vamos supor que só há restrições de desigualdade, $g(x)=1-x$, ou seja, $x \geq 1$. O minimo é encontrado em $\bar{x}=1$ e tem valor $f(1)=\frac{1}{3}$. A penalidade quadrática tem a forma

$$
P_{q}(x ; \beta)=\frac{1}{3} x^{3}+\beta\left\|(1-x)_{+}\right\|^{2} .
$$

Se $x \leq 1, P_{q}$ tem um mínimo local em $[0,1]: \bar{x}_{\beta}=-\beta+\sqrt{\beta^{2}+2 \beta}$ que converge para 1 se $\beta$ vai para infinito, mas $P_{q}$ vai para $-\infty$ quando $x \rightarrow-\infty$. Desse modo, o problema penalizado não admite mínimo.

Exemplo 2.3. Seja $f(x)=\frac{1}{3} x^{3}+\sin (x)$, com $x \in \mathbb{R}$. Tomamos $g(x)=-x$, ou seja, $x \geq 0$. $O$ mínimo é encontrado em $\bar{x}=0$ e tem valor $f(0)=0$. A penalidade fica

$$
P_{q}(x ; \beta)=\frac{1}{3} x^{3}+\sin (x)+\beta\left\|(-x)_{+}\right\|^{2} .
$$

$P_{q}(\cdot ; \beta)$ tem infinitos mínimos locais, em particular tomando a seqüência $\beta_{k}=k+\frac{\pi}{2}$ com $k$ inteiro positivo temos que $\bar{x}_{k}=-(2 k+1) \pi$ é solução e essa seqüência vai para $-\infty$ quando $k$ vai a infinito. Portanto, levando o parâmetro a infinito obtivemos uma seqüência de soluções que diverge.

Sem um algum tipo de controle no algoritmo do método de penalidades, a seqüência de soluções pode não existir ou não convergir para a solução do problema original.

\subsection{Penalidades Exatas Não-Diferenciáveis}

Com esse objetivo de obter um problema irrestrito que recupere a solução do problema original em uma única minimização, uma primeira tentativa de obter uma penalidade exata foi a de Zangwill [47, 46], ao criar a função

$$
\phi(x, \mu) \stackrel{\text { def }}{=} f(x)+\mu \max \left\{0, g_{1}(x), g_{2}(x), \ldots, g_{m}(x),\left|h_{1}(x)\right|,\left|h_{2}(x)\right|, \ldots,\left|h_{p}(x)\right|\right\} .
$$


Para verificarmos que em certos casos de fato podemos obter soluções do problema original minimizando $\phi$, teremos de introduzir brevemente alguns conceitos como dualidade e a função primal.

\subsubsection{Dualidade Lagrangiana}

Para facilitar as notações, admitiremos que $f, g$ e $h$ estão definidas em todo $\mathbb{R}^{n}$. Definimos o lagrangiano $L: \mathbb{R}^{n} \times \mathbb{R}^{m} \times \mathbb{R}^{p} \rightarrow \mathbb{R}$ do problema (NLP) como

$$
L(x, \lambda, \nu)=f(x)+\lambda^{T} g(x)+\nu^{T} h(x),
$$

em que $\lambda_{i}$ e $\nu_{j}$ são chamados de multiplicadores de Lagrange associados, respectivamente, às restrições $g_{i}(x) \leq 0$ e $h_{j}(x)=0$. Os vetores $\lambda$ e $\nu$ são chamados também de variáveis duais ou multiplicadores de Lagrange do problema (NLP).

A partir desse lagrangiano definimos também a função dual (lagrangiana) $q: \mathbb{R}^{m} \times \mathbb{R}^{p} \rightarrow \mathbb{R}$ como o mínimo do lagrangiano em $x$ para cada $\lambda$ e $\nu$ fixos:

$$
q(\lambda, \nu)=\min _{x \in \mathbb{R}^{n}} L(x, \lambda, \nu)=\min _{x}\left(f(x)+\sum_{i=1}^{m} \lambda_{i} g_{i}(x)+\sum_{j=1}^{p} \nu_{j} h_{j}(x)\right) .
$$

Podemos ter o lagrangiano ilimitado inferiormente em $x$, caso em que $q$ será $-\infty$. Como $q$ é em cada ponto um mínimo de funções afins em $(\lambda, \nu)$, é uma função côncava, independentemente de qualquer convexidade de $f, g$ ou $h$.

Uma propriedade importante da função dual é a sua capacidade de fornecer limites inferiores para a solução do problema original. De fato, suponha que $\tilde{x}$ é um ponto viável para o problema original e $\lambda \geq 0$. Então temos

$$
\sum_{i=1}^{m} \lambda_{i} g_{i}(\tilde{x})+\sum_{j=1}^{p} \nu_{j} h_{j}(\tilde{x}) \leq 0
$$

pois o segundo termo se anula e cada termo na primeira soma é o produto de um número positivo e um não positivo, e portanto

$$
L(\tilde{x}, \lambda, \nu)=f(\tilde{x})+\sum_{i=1}^{m} \lambda_{i} g_{i}(\tilde{x})+\sum_{j=1}^{p} \nu_{j} h_{j}(\tilde{x}) \leq f(\tilde{x}) .
$$


Desse modo

$$
q(\lambda, \nu)=\min _{x \in \mathbb{R}^{n}} L(x, \lambda, \nu) \leq L(\tilde{x}, \lambda, \nu) \leq f(\tilde{x})
$$

e como o valor ótimo $f^{*}$ do problema original pode ser aproximado por pontos viáveis, temos que

$$
q(\lambda, \nu) \leq f^{*}
$$

para todo $\lambda \geq 0$, o que efetivamente nos dá uma coleção de limites inferiores para o valor ótimo. A desigualdade acima é válida mas pode não trazer nenhuma informação no caso em que $q(\lambda, \nu)=-\infty$. Estaremos interessados exatamente nos valores de $\lambda \geq 0$ e $\nu$ em que $q$ tenha valores reais. Na verdade, gostaríamos de descobrir não só um limite inferior qualquer, mas o melhor limite inferior para o problema original que a função dual possa nos proporcionar. Para isso definimos o problema (lagrangiano) dual ao problema (NLP):

$$
\begin{gathered}
\max q(\lambda, \nu) \\
\text { s.a } \lambda \geq 0,
\end{gathered}
$$

e chamamos os pares $(\lambda, \nu) \operatorname{com} \lambda \geq 0$ para os quais $q$ é real de viáveis duais. Como $q$ é côncava e as restrições são convexas, temos que o problema dual é um problema de otimização convexo, independentemente do problema original ser convexo ou não.

Essa relação entre o valor ótimo dual, $q^{*}$, e o valor ótimo do problema original, $f^{*} \geq q^{*}$ é o teorema fraco de dualidade, ou dualidade fraca. Quando o problema original é convexo, muitas vezes temos um resultado mais importante, de que $f^{*}=q^{*}$. Dizemos que nesse caso vale a dualidade forte. Esse caso é de grande interesse pois permite obter a solução do problema original resolvendo-se o problema dual, o que pode ser mais fácil. Uma grande quantidade de métodos para resolver problemas de otimização beneficia-se da informação dual para auxiliar ou garantir a convergência a uma solução, entre eles os métodos de lagrangianos aumentados ou métodos de multiplicadores [40,39] e também as penalidades exatas diferenciáveis que estudaremos e desenvolveremos nesse trabalho.

A dualidade forte não vale para qualquer problema convexo, é necessário que as restrições satisfaçam uma condição de qualificação [7]. Um exemplo é a condição de Slater, que pede que exista um ponto estritamente viável, tal que as restrições de desigualdade sejam estritamente satisfeitas, ou seja, $\exists x$ tal que $h(x)=0$ e $g(x)<0$. Para problemas convexos, a condição de Slater é equivalente à uma outra condição de qualificação muito usada na literatura, a condição de Mangasarian-Fromovitz, que pede que os gradientes das restrições sejam 
linearmente independentes e exista uma direção $d \in \mathbb{R}^{n}$ tal que

$$
\begin{array}{rr}
\nabla h_{i}(x)^{T} d=0 & i=1 . . p \\
\nabla g_{i}(x)^{T} d<0 & \forall i \text { t.q. } g_{i}(x)=0 .
\end{array}
$$

Para funções não convexas a dualidade forte é muito mais rara, restrita a alguns casos especiais. Um estudo mais detalhado de dualidade e uma indicação geométrica dessa afirmação podem ser encontrados em diversos livros como $[9,12]$.

\subsubsection{Sensibilidade e a função primal}

Vamos agora tentar obter boas estimativas de como varia a função objetivo e as restrições próximo a uma solução do problema original.

Consideremos a seguinte variação perturbada do problema original

$$
\begin{array}{r}
\min f(x) \\
\text { s.a } g(x) \leq u \\
h(x)=v,
\end{array}
$$

em que afrouxamos (ou apertamos) a restrição de desigualdade e mudamos ligeiramente a restrição de igualdade. O caso em que $(u, v)=(0,0)$ recai no problema original, e estamos interessados em estudar a variação da solução do problema com essa perturbação nas restrições. Definimos então a função primal $p: \mathbb{R}^{m} \times \mathbb{R}^{p} \rightarrow \mathbb{R}$ por

$$
p(u, v)=\inf _{x}\{f(x)-g(x) \leq u \text { e } h(x)=v\}
$$

que nos dá o valor da solução do problema perturbado. Em particular temos $p(0,0)=f^{*}$. Não é difícil notar que no caso convexo, $p$ é convexa em $u$ e $v$ [12].

Vamos supor a partir de agora que nosso problema é mesmo convexo, que vale a dualidade forte e que o valor ótimo dual é atingido, o que é o caso por exemplo quando vale a condição de Slater.

Seja $(\bar{\lambda}, \bar{\nu})$ uma solução do problema dual. Pela definição da função dual temos que

$$
q(\bar{\lambda}, \bar{\nu}) \leq f(x)+\sum_{i=1}^{m} \bar{\lambda}_{i} g_{i}(x)+\sum_{j=1}^{p} \bar{\nu}_{j} h_{j}(x) .
$$


Se supusermos que $x$ é viável para o problema perturbado, lembrando que $\bar{\lambda} \geq 0$, temos também que

$$
f(x)+\sum_{i=1}^{m} \bar{\lambda}_{i} g_{i}(x)+\sum_{j=1}^{p} \bar{\nu}_{j} h_{j}(x) \leq f(x)+\sum_{i=1}^{m} \bar{\lambda}_{i} u_{i}+\sum_{j=1}^{p} \bar{\nu}_{j} v_{j} .
$$

Assim, como admitimos a dualidade forte, temos

$$
f^{*}=q(\bar{\lambda}, \bar{\nu}) \leq f(x)+\bar{\lambda}^{T} u+\bar{\nu}^{T} v
$$

Por fim, relembrando que a desigualdade acima é válida para todo ponto viável para o problema perturbado, usamos a definição de $p$ para ver que

$$
f^{*}-\bar{\lambda}^{T} u-\bar{\nu}^{T} v \leq p(u, v) .
$$

Essa relação nos dá uma boa medida da influência da modificação no valor ótimo quando as restrições do problema original são alteradas como no problema perturbado. De fato, a equação acima mostra que $-(\bar{\lambda}, \bar{\nu})$ é um subgradiente da função convexa $p$ na origem, e se $p$ for diferenciável, $-\bar{\lambda}$ e $-\bar{\nu}$ são suas derivadas parciais:

$$
\frac{\partial p}{\partial u_{i}}(0,0)=-\bar{\lambda}_{i} \text { e } \frac{\partial p}{\partial v_{j}}(0,0)=-\overline{\nu_{j}}
$$

o que é uma medida da taxa de variação do valor objetivo com a variação das restrições. Mesmo no caso em que $f(x)$ não é convexa, também temos que a diferenciabilidade de $p$ garante que $\nabla p(0,0)=-(\bar{\lambda}, \bar{\nu})[42]$.

Podemos agora aplicar esses resultados para verificar que a $\phi$ do início da seção é de fato uma penalidade exata.

\subsubsection{Penalidade Exata Não-Diferenciável}

Para facilitar a notação e as contas, nos restringiremos ao caso em que só há restrições de desigualdade, e continuamos supondo que o problema é convexo e vale a condição de Slater. Reescreveremos nosso problema então como

$$
\begin{aligned}
& \min f(x) \\
& \text { s.a } g(x) \leq 0
\end{aligned}
$$


com valor ótimo $f^{*}$ e construiremos o problema auxiliar

$$
\min _{x} \phi(x, \mu) \stackrel{\text { def }}{=} f(x)+\mu \max \left\{0, g_{1}(x), g_{2}(x), \ldots, g_{m}(x)\right\},
$$

com valor ótimo $\tilde{f}^{*}$. Como máximos e somas entre funções convexas são convexos e $\mu>0, \phi$ é convexo. Além disso, podemos reescrever o problema $\min _{x} \phi(x, \mu)$ como

$$
\begin{array}{ll}
\min & f(x)+\mu y \\
\text { s.a } & g_{i}(x) \leq y \text { para } i=1 . . m \\
& y \geq 0 .
\end{array}
$$

A equivalência das soluções dos dois problemas será obtida ao olharmos para os duais. A função dual do problema original (2.4) é

$$
q(\lambda)=\inf _{x} L(x, \lambda)=\inf _{x}\left(f(x)+\lambda^{T} g(x)\right)
$$

e o problema dual é

$$
\begin{aligned}
& \max q(\lambda) \\
& \text { s.a } \lambda \geq 0 .
\end{aligned}
$$

Já a função dual do problema (2.5) é

$$
\begin{aligned}
\tilde{q}\left(\lambda, \lambda_{0}\right) & =\inf _{x, y} \tilde{L}\left(x, y, \lambda, \lambda_{0}\right)=\inf _{x, y}\left(f(x)+\mu y+\sum_{i=1}^{m} \lambda_{i}\left(g_{i}(x)-y\right)-\lambda_{0} y\right) \\
& =\inf _{x}\left(f(x)+\lambda^{T} g(x)\right)+\inf _{y}\left(y\left(\mu-\sum_{i=0}^{m} \lambda_{i}\right)\right) \\
& =q(\lambda)+\left\{\begin{aligned}
0, & \text { se } \mu=\sum_{i=0}^{m} \lambda_{i} \\
-\infty, & \text { caso contrário }
\end{aligned}\right. \\
& = \begin{cases}q(\lambda), & \text { se } \mu=\sum_{i=0}^{m} \lambda_{i} \\
-\infty, & \text { caso contrário. }\end{cases}
\end{aligned}
$$

Portanto o problema dual a (2.5) é

$$
\begin{aligned}
& \max \tilde{q}\left(\lambda, \lambda_{0}\right) \\
& \quad \text { s.a }\left(\lambda, \lambda_{0}\right) \geq 0,
\end{aligned}
$$


que é equivalente, fazendo $\lambda_{0}=\mu-\sum_{i=1}^{m} \lambda_{i}$, a

$$
\begin{array}{ll}
\max & q(\lambda) \\
\text { s.a } \lambda \geq 0 & \\
\sum_{i=1}^{m} \lambda_{i} \leq \mu .
\end{array}
$$

Como vale a condição de Slater para o problema (2.4), ela também valerá para o problema (2.5) e ambos terão dualidade forte. Portanto, se o valor ótimo dual do problema original é atingido para um $\bar{\lambda}$ e se temos $\mu \geq \sum_{i=1}^{m} \bar{\lambda}_{i}$, o valor ótimo dual do problema auxiliar será atingido e será o mesmo. A dualidade forte nos dará

$$
\tilde{f}^{*}=\tilde{q}\left(\bar{\lambda}, \bar{\lambda}_{0}\right)=q(\bar{\lambda})=f^{*}
$$

a igualdade que buscávamos.

Uma solução $\bar{x}$ de (2.4) é por definição viável para o problema original, e também viável para o problema auxiliar, pois esse está definido em todo $\mathbb{R}^{n}$. De fato, ela será solução do problema auxiliar, pois para $\mu \geq \sum_{i=1}^{m} \bar{\lambda}_{i}$, vem direto da definição de $\phi$ que $\phi(\bar{x}, \mu)=$ $f(\bar{x})+0=f^{*}=\tilde{f}^{*}$.

Especificamente nesse caso podemos verificar também que as soluções $\tilde{x}$ do problema auxiliar também são necessariamente soluções do problema original. Já sabemos que se $\mu \geq \sum_{i=1}^{m} \bar{\lambda}_{i}$, os valores ótimos do problema original e do problema auxiliar são os mesmos. Veremos agora que se $\tilde{x}$ é uma solução do problema auxiliar e exigirmos a condição levemente mais forte que $\mu>\sum_{i=1}^{m} \bar{\lambda}_{i}$, ele também será viável para o problema original e nesse caso, a igualdade dos valores ótimos implicará que $\tilde{x}$ será ótimo para o problema (2.4).

Efetivamente, dada uma solução ótima do problema auxiliar, $\tilde{x}$, se tal solução fosse inviável, teríamos $\phi(\tilde{x}, \mu)=f(\tilde{x})+\mu g_{k}(\tilde{x})=\tilde{f}^{*}$ para um certo índice $k, \operatorname{com} g_{k}(\tilde{x}) \geq g_{i}(\tilde{x})$ para todo $i$ de 1 a $m$. Nesse caso, como $\bar{\lambda} \geq 0$, temos

$$
L(\tilde{x}, \bar{\lambda})=f(\tilde{x})+\bar{\lambda}^{T} g(\tilde{x}) \leq f(\tilde{x})+\left(\sum_{i=1}^{m} \bar{\lambda}_{i}\right) g_{k}(\tilde{x}) .
$$

Da definição da função dual e como também vale que $g_{k}(\tilde{x}) \geq 0$, vem então que

$$
q(\bar{\lambda}) \leq L(\tilde{x}, \bar{\lambda}) \leq f(\tilde{x})+\left(\sum_{i=1}^{m} \bar{\lambda}_{i}\right) g_{k}(\tilde{x})<f(\tilde{x})+\mu g_{k}(\tilde{x})=\tilde{f}^{*}=q(\bar{\lambda}) .
$$


Um absurdo. Portanto não pode existir solução do problema auxiliar inviável para o problema original, e toda solução de um dos problemas é também solução do outro.

\subsubsection{Definições de Exatidão}

É importante ressaltar que resultados análogos podem ser obtidos em casos mais gerais, com restrições de igualdade presentes no problema original e com hipóteses mais fracas sobre as funções. Em particular é possível relaxar as hipóteses de convexidade e usar outras condições de qualificação, como a condição de regularidade, que exige os gradientes das restrições sejam linearmente independentes.

Essas generalizações, que podem ser encontradas em trabalhos como [10, 47, 36, 28], exigem que a análise da penalidade exata apresentada acima seja mais cuidadosa. Em especial, o resultado mais comum nesse contexto mais geral é que soluções do problema original são soluções do problema penalizado, mas não há garantias de que o problema penalizado não possua outras soluções que não sejam ótimos do problema original. O estudo de quando isso ocorre, ou de possíveis situações intermediárias levou à definição de uma série de possíveis níveis de exatidão para uma penalidade, que mencionaremos brevemente aqui. Um estudo mais detalhado pode ser feito por meio de $[16,28]$.

As penalidade são avaliadas principalmente quanto à equivalência entre soluções do problema original e do problema penalizado em caráter local e global.

A grosso modo, uma penalidade exata para um problema de minimização $\mathcal{P}_{0}$ é uma função $P(\cdot, c)$ tal que para uma escolha apropriada do parâmetro de penalização $c$, a sua minimização resulta em uma solução de $\mathcal{P}_{0}$. Espera-se também que a escolha do parâmetro possa ser feita de modo que, por exemplo, essa propriedade seja verdadeira para todo $c$ acima de um certo limiar $c\left(\mathcal{P}_{0}\right)$ que dependa do problema dado.

Dada uma penalidade $P$, a existência de parâmetros $c$ para qualquer problema, em particular os não convexos, só pode ser obtida se restringirmos a minimização a compactos. Como vimos no exemplo (2.2), mesmo em casos simples e com restrições convexas podemos ter uma penalidade que não possua mínimos. A única maneira de garantir que existe um parâmetro de penalização finito é restringindo a minimização a um compacto; o parâmetro garantirá que mínimos espúrios só poderão estar fora deste compacto. Essa restrição pode não ser necessária para garantir que alguns mínimos do problema original sejam mínimos do problema penalizado, mas é essencial para obter o resultado contrário e mais relevante do ponto de vista das aplicações, ou seja, de que ao resolver-se o problema penalizado recupera-se a solução do 
problema original.

Dado então um compacto $\mathcal{D}$, teremos dois problemas-chave,

$$
\min _{x \in \mathcal{D} \cap \mathcal{F}} f(x)
$$

onde $\mathcal{F}$ é o conjunto viável original, e o problema penalizado

$$
\min _{x \in \mathcal{D}} P(x, c)
$$

Os conjuntos das soluções locais e globais de cada problema serão denotadas por $\mathcal{L}_{\mathcal{P}}, \mathcal{G}_{\mathcal{P}}$, $\mathcal{L}_{\mathcal{Q}}(c)$ e $\mathcal{G}_{\mathcal{Q}}(c)$, respectivamente. Podemos apresentar as definições.

Definição 2.4. $P(x, c)$ é dita uma penalidade fracamente exata para o problema $\mathcal{P}$ com relação a $\mathcal{D}$, se existe $\bar{c}$ tal que $\mathcal{G}_{\mathcal{P}}=\mathcal{G}_{\mathcal{Q}}(c)$ para $c>\bar{c}$.

Em particular, se todas as soluções globais do problema original estiverem em $\mathcal{D}$, podemos obtê-las simplesmente resolvendo globalmente $\mathcal{Q}$. A fraqueza da definição está exatamente na necessidade de achar mínimos globais, coisa que a maioria dos algoritmos de minimização irrestrita não tem como garantir na ausência de convexidade. É importante relembrar que mesmo que $f$ seja convexa, $P$ em geral não será.

Definição 2.5. $P(x, c)$ é dita uma penalidade exata para o problema $\mathcal{P}$ com relação a $\mathcal{D}$, se existe $\bar{c}$ tal que $\mathcal{G}_{\mathcal{P}}=\mathcal{G}_{\mathcal{Q}}(c)$ e $\mathcal{L}_{\mathcal{Q}}(c) \subset \mathcal{L}_{\mathcal{P}}$ para $c>\bar{c}$.

Não é necessariamente essencial que a cada solução local de $\mathcal{P}$ tenhamos uma correspondente em $\mathcal{Q}$, contanto que ao minimizarmos esse, agora sim localmente, encontremos soluções locais do nosso problema de interesse $\mathcal{P}_{0}$. Principalmente se levarmos em conta que a equivalência das soluções globais ainda é um requisito. Mesmo assim em alguns casos temos também o seguinte

Definição 2.6. $P(x, c)$ é dita uma penalidade fortemente exata para o problema $\mathcal{P}$ com relação a $\mathcal{D}$, se existe $\bar{c}$ tal que $\mathcal{G}_{\mathcal{P}}=\mathcal{G}_{\mathcal{Q}}(c), \mathcal{L}_{\mathcal{Q}}(c) \subset \mathcal{L}_{\mathcal{P}}$ e $\mathcal{L}_{\mathcal{P}} \cap$ int $\mathcal{D} \subset \mathcal{L}_{\mathcal{Q}}(c)$ para $c>\bar{c}$.

Esse resultado dá a equivalência total de soluções a menos da fronteira do conjunto $\mathcal{D}$. Há ainda definições que pedem condições para que seqüências de mínimos locais de Q não convirjam para a fronteira de $\mathcal{D}$. Remetemos o leitor interessado às referências já citadas.

Na prática, não se pode determinar facilmente qual o parâmetro de penalização para um dado problema e dado compacto. Por esse motivo, em geral os algoritmos, mesmo se tratando 
de uma penalidade exata, resolvem aproximadamente o problema penalizado e verificam a solução, acertando o parâmetro e refazendo a minimização, o que resulta numa seqüência que convergirá a uma solução. Na prática, entretanto, os algoritmos para penalidade exatas convergem após muito poucas atualizações do parâmetro de penalização e efetivamente resolvem o problema em poucos passos [27, 14].

\subsection{Primeiras Penalidades Exatas Diferenciáveis: Restrições de Igualdade}

Apesar de não ser a primeira penalidade exata a surgir na literatura, pois Fletcher já havia apresentado um método em 1970 [23, 26, 24], a formulação de Di Pillo e Grippo para problemas com restrições de igualdade $[14,17]$ nos ajudará a entender melhor o caminho para se chegar à extensão de penalidade exata para problemas de desigualdades variacionais que será proposta no próximo capítulo. Veremos que tanto a versão de Fletcher, quanto a sua revisão por Mukai e Polak [32, 31], são casos particulares da penalidade proposta pelos italianos.

Para facilitar a notação, iremos inicialmente tratar do problema somente com restrições de igualdade, ou seja,

$$
\begin{array}{cl}
\min & f(x) \\
\text { s.a } & h(x)=0,
\end{array}
$$

em que $f: \mathbb{R}^{n} \rightarrow \mathbb{R}$ e $h: \mathbb{R}^{n} \rightarrow \mathbb{R}^{m}$ são duas vezes continuamente diferenciáveis. Utilizaremos também o lagrangiano usual

$$
L(x, \lambda)=f(x)+\lambda^{T} h(x) .
$$

A partir deste lagrangiano temos as conhecidas condições necessária de otimalidade de primeira ordem

$$
\left[\begin{array}{c}
\nabla_{x} L(\bar{x}, \bar{\lambda}) \\
\nabla_{\lambda} L(\bar{x}, \bar{\lambda})
\end{array}\right]=0 .
$$

Uma estratégia possível para se resolver o problema (2.9) é tentar resolver o problema acima, repensando-o como um problema de mínimos quadrados

$$
\min _{x, \lambda}\left\|\nabla_{x} L(x, \lambda)\right\|^{2}+\|h(x)\|^{2}
$$

Tal formulação não distingue, por exemplo, entre máximos e mínimos de problema original. Mas é inspirada por ela que Di Pillo e Grippo adicionam ao lagrangiano aumentado clássico 
$L_{A}(x ; \lambda)=f(x)+\lambda^{T} h(x)+c\|h(x)\|^{2}$ um termo a mais, penalizando a violação da condição de primeira ordem na derivada com relação a $x$ :

$$
w(x, \lambda ; c)=w_{c}(x, \lambda)=f(x)+\lambda^{T} h(x)+\frac{c}{2}\|h(x)\|^{2}+\frac{1}{2}\left\|M(x) \nabla_{x} L(x, \lambda)\right\|^{2} .
$$

$M(\cdot)$ é uma matriz de ponderação $p \times n$ com $m \leq p \leq n$ duas vezes diferenciável que é a chave para a equivalência entre essa e as outras penalidade exatas diferenciáveis.

Para facilitar o trabalho à frente vale a pena explicitar as derivadas parciais de $w$

$$
\begin{array}{lc}
\nabla_{\lambda} w_{c}(x, \lambda)= & h(x)+\nabla h(x) M(x)^{T} M(x) \nabla_{x} L(x, \lambda) \\
\nabla_{x} w_{c}(x, \lambda)= & \nabla f(x)+\nabla h(x)^{T} \lambda+c \nabla h(x)^{T} h(x) \\
& +\nabla_{x x}^{2} L(x, \lambda) M(x)^{T} M(x) \nabla_{x} L(x, \lambda) \\
& +\left(\sum_{j=1}^{p}\left[\nabla m_{j}(x) \nabla_{x} L(x, \lambda) e_{j}^{T}\right]\right) M(x) \nabla_{x} L(x, \lambda) .
\end{array}
$$

Nas fórmulas acima e daqui em diante $\nabla h(x)$ é a matriz cujas linhas são os gradientes das componentes de $h, e_{j}$ são os versores da base canônica de $\mathbb{R}^{p}$ e $m_{j}(x)$ é a $j$-ésima linha de $M$, portanto $M(x)=\sum_{j=1}^{p} e_{j} m_{j}(x)$. A diferenciabilidade de todas as funções envolvidas implica na diferenciabilidade dessa penalidade.

A seguir, vamos mostrar alguns resultados iniciais que são característicos do que se encontra na literatura de penalidades exatas diferenciáveis. Inicialmente observamos a relação entre pontos estacionários de $L$ e $w_{c}$. Assumiremos para facilitar que $M$ é $m \times n$. No caso geral $p>m$ podemos trocar a hipótese de não-singularidade de $M \nabla h$ pela exigência de posto máximo.

Proposição 2.7. Se $(\bar{x}, \bar{\lambda})$ é estacionário de $L$, também é estacionário de $w_{c}, \forall c>0$ e vale ainda que $w_{c}(\bar{x}, \bar{\lambda})=f(\bar{x})$.

Demonstração. É imediato das fórmulas acima que se $\nabla_{x} L(\bar{x}, \bar{\lambda})=0$ e $\nabla_{\lambda} L(\bar{x}, \bar{\lambda})=h(\bar{x})=0$, ambas derivadas parciais de $w_{c}$ se anulam em $(\bar{x}, \bar{\lambda})$. A igualdade de valores vem direto do anulamento dos termos na definição de $w_{c}$.

Vale também um resultado no outro sentido.

Proposição 2.8. Para $\forall c>0$, se $(\bar{x}, \bar{\lambda})$ é estacionário para $w_{c} \operatorname{com} M(\bar{x}) \nabla h(\bar{x})^{T}$ não-singular e $h(\bar{x})=0$, então $(\bar{x}, \bar{\lambda})$ é estacionário para $L$ e $w_{c}(\bar{x}, \bar{\lambda})=f(\bar{x})$. 
Demonstração. Se $(\bar{x}, \bar{\lambda})$ é estacionário de $w_{c}, \nabla_{\lambda} w_{c}$ se anula e substituindo na sua fórmula as hipóteses temos $\nabla h(\bar{x}) M(\bar{x})^{T} M(\bar{x}) \nabla_{x} L(\bar{x}, \bar{\lambda})=0 \quad \Longrightarrow \quad M(\bar{x}) \nabla_{x} L(\bar{x}, \bar{\lambda})=0$ que, substituído junto com $h(\bar{x})=0$ em $\nabla_{x} w_{c}(\bar{x}, \bar{\lambda})=0$ dá $\nabla_{x} L(\bar{x}, \bar{\lambda})=0$. Lembrando novamente que $h(\bar{x})=0$, temos a tese. Para a igualdade dos valores funcionais basta, como anteriormente, notar os termos que se anulam na definição de $w_{c}$.

Outro resultado típico vai nos livrar da hipótese explícita de viabilidade de $\bar{x}$, compensando com o parâmetro $c$. Mas para isso teremos de restringir nossa minimização a um compacto.

Proposição 2.9. Seja $\Omega=X \times \Lambda$ um compacto de $\mathbb{R}^{n} \times \mathbb{R}^{m}$ tal que $M(x) \nabla h(x)^{T}$ é nãosingular para todo $x$ em $X$. Então existe $\tilde{c}$ tal que se $c \geq \tilde{c} e(\bar{x}, \bar{\lambda}) \in \Omega$ é estacionário para $w_{c}$, então $(\bar{x}, \bar{\lambda})$ é estacionário para $L$ e $w_{c}(\bar{x}, \bar{\lambda})=f(\bar{x})$.

Demonstração. Igualando a expressão de $\nabla_{\lambda} w_{c}$ a 0 , temos $M(\bar{x}) \nabla_{x} L(\bar{x}, \bar{\lambda})=-\left[\nabla h(\bar{x}) M(\bar{x})^{T}\right]^{-1} h(\bar{x})$. Definindo agora $Q(x) \stackrel{\text { def }}{=}-\left[\nabla h(x) M(x)^{T}\right]^{-1}$ e aplicando $M(\bar{x})$ a $\nabla_{x} w_{c}(\bar{x}, \bar{\lambda})=0$ temos

$$
\begin{aligned}
0 & =M \nabla_{x} L+c M \nabla h^{T} h+M\left(\nabla_{x x}^{2} L M^{T}+\sum \nabla m_{j} \nabla_{x} L e_{j}^{T}\right) M \nabla_{x} L \\
& =\quad\left[Q+c M \nabla h^{T}-M\left(\nabla_{x x}^{2} L M^{T}+\sum \nabla m_{j} \nabla_{x} L e_{j}^{T}\right) Q\right] h .
\end{aligned}
$$

Nas expressões acima todas as função estão calculadas em $\bar{x}$ ou $(\bar{x}, \bar{\lambda})$. Podemos escrever que $[c A(\bar{x})+B(\bar{x}, \bar{\lambda})] h(\bar{x})=0$ com $B$ contínua em $\Omega$ e $A$ não-singular.

Pela desigualdade triangular obtemos, tomando um $z$ qualquer em $\mathbb{R}^{m}$,

$$
\|[c A(x)+B(x, \lambda)] z\| \geq c\|A(x) z\|-\|B(x, \lambda) z\| .
$$

Podemos supor, sem perda de generalidade, que $z$ é um versor, ou seja, tem norma 1. Pela continuidade de $B$ e pelas compacidades de $\Omega$ e da esfera de raio unitário, o problema

$$
\begin{gathered}
\max \|B(x, \lambda) z\| \\
\text { s.a }(x, \lambda) \in \Omega \\
\|z\|=1,
\end{gathered}
$$

atinge um máximo finito que chamaremos de $\alpha$. Temos então que

$$
\|[c A(x)+B(x, \lambda)] z\| \geq c\|A(x) z\|-\alpha .
$$

Mas $\|A(x) z\|$ é estritamente positiva em $X$. De fato, se tivéssemos uma seqüência $\left(x^{k}, z^{k}\right)$ 
com $x^{k} \in X$ e $z^{k}$ na esfera de raio unitário tal que $\left\|A\left(x^{k}\right) z^{k}\right\| \rightarrow 0$, a compacidade nos daria uma subseqüência tal que $\left(x^{k}, z^{k}\right) \rightarrow(\tilde{x}, \tilde{z})$ e pela continuidade de $A(\cdot)$, teríamos $A(\tilde{x}) \tilde{z}=0$ $\operatorname{com} \tilde{z} \neq 0$ o que contrariaria a não-singularidade de $A$ em todo $X$, absurdo. Portanto $\exists \varepsilon>0$ tal que $\|A(x) z\|>\varepsilon$ e basta tomarmos $\tilde{c}>\frac{\alpha}{\varepsilon}$ para termos

$$
\|[\tilde{c} A(x)+B(x, \lambda)] z\|>0
$$

para todo $(x, \lambda)$ em $\Omega$ e $z$ versor. Na verdade o resultado também é válido para todo $z$ não nulo, pois podemos sempre dividir ambos os lados pela sua norma.

Olhando novamente para a equação $[c A(\bar{x})+B(\bar{x}, \bar{\lambda})] h(\bar{x})=0$, acabamos de garantir que $h(\bar{x})=0$ e recai-se na proposição anterior.

Esse é o típico resultado de penalidades exatas diferenciáveis, que pode ser melhorado para dar equivalência entre mínimos do problema inicial e de $w$, um resultado mais fino do que a simples equivalência entre pontos estacionários. Porém, tais resultados dependerão diretamente de operações sobre os valores funcionais que não podem ser feitas no contexto de desigualdades variacionais e não nos serão úteis no restante do trabalho. O leitor interessado poderá consultar [14].

\subsubsection{Eliminando os multiplicadores}

Até agora a função a ser minimizada não é de fato uma penalidade e sim um lagrangiano aumentado exato pois ele depende também das variáveis duais. Para transformá-la numa penalidade, vamos remover as variáveis duais através de uma escolha apropriada da matriz $M$. Através desse modelo iremos, saindo da formulação de Di Pillo e Grippo, obter a penalidade original de Fletcher.

As próprias hipóteses das proposições acima já induzem a uma escolha de $M$ que garanta que $M(x) \nabla h(x)^{T}$ seja não-singular. Se queremos nos livrar dessa condição da maneira mais simples, bastaria a igualarmos à matriz identidade. Queremos escolher

$$
M(x)=\left[\nabla h(x) \nabla h(x)^{T}\right]^{-1} \nabla h(x) .
$$

Convém notar, entretanto, que a matriz entre colchetes só terá inversa, e portanto essa $M(x)$ só estará bem definida, no caso em que $\nabla h(x)$ tem posto máximo, em que dizemos que $x$ é um ponto regular. Assumiremos aqui que essa hipótese é verdadeira. 
Temos assim

$$
w_{c}(x, \lambda)=f(x)+\lambda^{T} h(x)+\frac{c}{2}\|h(x)\|^{2}+\frac{1}{2}\|M(x) \nabla f(x)+\lambda\|^{2} .
$$

No último termo, é exatamente a identidade que aparece multiplicando $\lambda$. A função acima é quadrática convexa em $\lambda$. Ao minimizarmos $w_{c}$ em todo espaço podemos encontrar explicitamente o $\lambda$ correspondente. Conseguimos desacoplar a minimização em cada variável. Para um $x$ fixo temos que o mínimo é encontrado derivando-se com relação a $\lambda$ e igualando a zero

$$
0=h(x)+\lambda+M(x) \nabla f(x) .
$$

Isolando $\lambda$, podemos na verdade definir uma função que, para cada $x$, calcula o $\lambda$ correspondente para que o mínimo seja atingido

$$
\hat{\lambda}(x)=-h(x)-M(x) \nabla f(x)
$$

A minimização de $w_{c}$ pode ser feita automaticamente em $x$ e $\lambda$, bastando calcular $\hat{\lambda}(x)$ diretamente. Substituindo $\hat{\lambda}(\cdot)$ em $w_{c}$ chegamos a

$$
\begin{aligned}
\hat{w}_{c}(x)=w_{c}(x, \hat{\lambda}(x))= & f(x)+\hat{\lambda}(x)^{T} h(x)+\frac{c}{2}\|h(x)\|^{2}+\frac{1}{2}\|M(x) \nabla f(x)+\hat{\lambda}(x)\|^{2} \\
= & \\
=\quad & f(x)-\|h(x)\|^{2}-(M(x) \nabla f(x))^{T} h(x)+\frac{c}{2}\|h(x)\|^{2}+\|h(x)\|^{2} \\
= &
\end{aligned}
$$

Tal escolha de $M$ também nos dá a manutenção da diferenciabilidade. Ela permite o cálculo da penalidade sem o uso de variáveis extras. A desvantagem é que torna-se necessário fazer uma inversão de matriz para podermos avaliar a função. Mas tal inversão, dependendo do método usado para resolver o problema em duas variáveis, já teria de aparecer em outra etapa do processo.

É importante notar a semelhança dessa função com o próprio lagrangiano aumentado clássico. Na verdade a substituição

$$
\lambda(x)=-M(x) \nabla f(x)
$$

nos recupera a forma $\hat{w}_{c}(x)=f(x)+\lambda(x)^{T} h(x)+\frac{c}{2}\|h(x)\|^{2}$. Vamos explorar um pouco essa idéia na próxima seção. 


\subsubsection{A relação com métodos de multiplicadores}

Ao resolver um problema de minimização por um método de lagrangianos aumentados ou método de multiplicadores, resolvemos iterativamente o problema

$$
\min _{x} L_{A}^{k}(x)=f(x)+\lambda_{k}^{T} h(x)+\frac{c_{k}}{2}\|h(x)\|^{2}
$$

com atualização de $\lambda_{k}$ a cada passo e possivelmente do parâmetro $c_{k}$ também. O grande interesse em tais métodos está no fato de que, com uma atualização adequada de $\lambda_{k}$, a seqüência $\left\{\bar{x}^{k}\right\}$ de soluções pode convergir a uma solução do problema original mesmo sem ter que levar $c_{k}$ a infinito, diferentemente do que ocorre com as penalidades clássicas. É exatamente dessa idéia que surgem muitos dos métodos de penalidades exatas diferenciáveis. Ao tentar incorporar a escolha do multiplicador $\lambda$ diretamente na função, busca-se obter um lagrangiano aumentado que só dependa das variáveis primais e que permita recuperar uma solução do problema original através de uma única minimização.

Foi essa a idéia de Fletcher e que foi explorada por Mukai e Polak. A escolha de $\lambda$ foi novamente motivada pela tentativa de satisfazer as condições necessárias de primeira ordem. Definiu-se

$$
\hat{\lambda}(x)=\arg \min _{\lambda}\left\|\nabla_{x} L(x, \lambda)\right\|^{2} .
$$

Mas lembrando que $\nabla_{x} L(x, \lambda)=\nabla f(x)+\lambda^{T} \nabla h(x)$, o mínimo é o de uma função quadrática convexa e pode ser encontrado derivando: $0=\nabla h(x) \nabla h(x)^{T} \lambda+\nabla h(x) \nabla f(x)$ e finalmente $\hat{\lambda}(x)=-\left[\nabla h(x) \nabla h(x)^{T}\right]^{-1} \nabla h(x) \nabla f(x)$. É a mesma escolha de multiplicador que (2.11), mostrando a ligação entre os métodos de Di Pillo e Grippo e Mukai e Polak.

Essa estratégia de escolher um bom multiplicador e incorporar sua escolha no lagrangiano aumentado também pose ser usada para se obter penalidades exatas no caso de restrições de desigualdades.

\subsection{Penalidades Exatas para Desigualdades}

A primeira penalidade exata diferenciável para problemas de desigualdades surgiu novamente com Fletcher, em 1973 [25]. Essa penalidade vinha diretamente de sua formulação anterior para problemas de igualdade. Ao notar que tudo se passava como se fossem calculados os multiplicadores reais de uma linearização das restrições, a mesma estratégia foi usada para problemas de desigualdade, calculando-se os multiplicadores de um problema que fazia uma 
aproximação quadrática da função objetivo e aproximações lineares das restrições. O problema de minimização não tinha de ser resolvido e era possível calcular os multiplicadores diretamente, mas tal penalidade tinha problemas de não-diferenciabilidade, o que causava problemas para muitos algoritmos.

Em 1979, Glad e Polak retomaram as idéias de Fletcher e melhoraram as estimativas dos multiplicadores para garantir que a diferenciabilidade se desse em todo o espaço [27]. Além disso, no algoritmo presente no mesmo artigo é incorporada uma estratégia semelhante a de Mukai e Polak para controlar o aumento do parâmetro de penalização. Porém, a estratégia proposta ainda apresenta limitações, como a necessidade de se calcular as segundas derivadas das restrições para a obtenção dos gradientes da penalidade a cada passo. Ainda, a convergência só é provada para o caso em que se garante que todos os pontos KKT tem complementaridade estrita e hessiana do lagrangiano positiva definida.

Outra forma de lidar com desigualdades é uma adaptação do lagrangiano aumentado exato de Di Pillo e Grippo. As restrições de desigualdade são transformadas em restrições de igualdade por meio de variáveis auxiliares, e com uma nova escolha apropriada da matriz de ponderação $M$ podemos isolar essas variáveis auxiliares na minimização. Isso permite definir, assim como fizemos com $\hat{\lambda}$, uma função que calcule diretamente as variáveis de folga a partir de $x$ e $\lambda$. O problema dessa estratégia é que as variáveis duais não podem mais ser facilmente retiradas e a função resultante ainda é não-diferenciável em alguns pontos.

Vamos ver brevemente a adaptação feita por Di Pillo e Grippo para que seu lagrangiano aumentado exato também pudesse cuidar de restrições de desigualdade. Para simplificar a notação, vamos supor que não há mais restrições de igualdade. Nosso problema é agora

$$
\begin{array}{cl}
\min & f(x) \\
\text { s.a } & g(x) \leq 0 .
\end{array}
$$

Para resolvermos esse problema com a penalidade anterior, iremos criar variáveis artificiais novas, $z$, transformando cada restrição em uma nova, de igualdade. Supondo $g: \mathbb{R}^{n} \rightarrow \mathbb{R}^{m}$ vamos substituir nosso problema por

$$
\begin{array}{cl}
\min & \hat{f}(x, z)=f(x) \\
\text { s.a } & \hat{h}_{j}(x, z)=g_{j}(x)+z_{j}^{2}=0 j=1 \ldots m .
\end{array}
$$


Temos então

$$
\begin{aligned}
w_{c}(x, z, \lambda) & =\hat{f}(x, z)+\sum_{j=1}^{m}\left[\lambda_{j}\left(g_{j}(x)+z_{j}^{2}\right)+\frac{c}{2}\left(g_{j}(x)+z_{j}^{2}\right)^{2}\right] \\
& +\frac{1}{2}\left\|\tilde{M}(x, z)\left(\nabla_{x, z} \hat{f}(x)+\nabla_{x, z} \hat{h}(x, z)^{T} \lambda\right)\right\|^{2}
\end{aligned}
$$

Nesse caso, $\tilde{M}$ e $\nabla \hat{h}$ são matrizes $m \times(n+m)$. Vamos reescrever o último termo para facilitar a visualização.

$$
\begin{aligned}
& \tilde{M}(x, z)\left(\nabla_{x, z} \hat{f}(x)+\nabla_{x, z} \hat{h}(x, z)^{T} \lambda\right)= \\
& \tilde{M}(x, z)\left(\left[\begin{array}{c}
\nabla_{x} \hat{f}(x, z) \\
\nabla_{z} \hat{f}(x, z)
\end{array}\right]+\left[\begin{array}{ccc}
\nabla_{x} \tilde{h}_{1}(x, z) & \ldots & \nabla_{x} \tilde{h}_{m}(x, z) \\
\nabla_{z} \tilde{h}_{1}(x, z) & \ldots & \nabla_{z} \tilde{h}_{m}(x, z)
\end{array}\right] \lambda\right)= \\
& {\left[\begin{array}{ll}
\tilde{M}_{1(m \times n)}(x, z) & \tilde{M}_{2(m \times m)}(x, z)
\end{array}\right]\left(\left[\begin{array}{c}
\nabla f(x) \\
0
\end{array}\right]+\left[\begin{array}{ccc}
\nabla g_{1}(x) & \ldots & \nabla g_{m}(x) \\
z_{1} e_{1} & \ldots & z_{m} e_{m}
\end{array}\right] \lambda\right)=} \\
& {\left[\begin{array}{c}
\tilde{M}_{1}(x, z)\left(\nabla f(x)+\nabla g(x)^{T} \lambda\right) \\
\tilde{M}_{2}(x, z) Z \lambda
\end{array}\right] \text {. }}
\end{aligned}
$$

Na última expressão $Z$ denota a matriz diagonal construída a partir de $z$. Como pretendemos isolar as variáveis de folga $z$, é interessante assumir que $M$ é da forma $\tilde{M}_{1}(x, z)=M(x)$ e $\tilde{M}_{2}(x, z)=\hat{M}(z)$. Voltando à penalidade temos

$$
\begin{aligned}
& w_{c}(x, z, \lambda)=f(x)+\sum_{j=1}^{m}\left[\lambda_{j} g_{j}(x)+\frac{c}{2}\left(g_{j}(x)\right)^{2}\right]+\sum_{j=1}^{m}\left[\left(\lambda_{j}+c g_{j}(x)\right) z_{j}^{2}+\frac{c}{2} z_{j}^{4}\right] \\
& +\frac{1}{2}\left\|\tilde{M}(x, z)\left(\nabla_{x, z} \hat{f}(x)+\nabla_{x, z} \hat{h}(x, z)^{\prime} \lambda\right)\right\|^{2} \\
& =f(x)+\lambda^{T} g(x)+\frac{c}{2}\|g(x)\|^{2}+\sum_{j=1}^{m}\left[\left(\lambda_{j}+c g_{j}(x)\right) z_{j}^{2}+\frac{c}{2} z_{j}^{4}\right] \\
& +\frac{1}{2}\left\|\left[\begin{array}{c}
\tilde{M}_{1}(x, z)\left(\nabla f(x)+\nabla g(x)^{T} \lambda\right) \\
\tilde{M}_{2}(x, z) Z \lambda
\end{array}\right]\right\|^{2} \\
& =L(x, \lambda)+\frac{c}{2}\|g(x)\|^{2}+z^{T} Z^{T}(\lambda+c g(x))+\frac{c}{2} z^{T} Z^{T} Z z \\
& +\frac{1}{2}\left\|M(x) \nabla_{x} L(x, \lambda)\right\|^{2}+\frac{1}{2}\|\hat{M}(z) Z \lambda\|^{2} .
\end{aligned}
$$


Assumiremos agora também que $\hat{M}(z)$ é diagonal constante, obtendo com isso

$$
\|\hat{M}(z) Z \lambda\|^{2}=\sum_{j=1}^{m} m_{j j}^{2} \lambda_{j}^{2} z_{j}^{2} .
$$

Podemos observar que $w_{c}$ é quadrática convexa em $z^{2}$ e podemos minimizá-la explicitamente em $z$. Reescrevendo $z_{j}^{2}=u_{j}$ temos que cada coordenada deve ser escolhida em

$$
\arg \min _{u_{j}} u_{j}\left(\lambda_{j}+c g_{j}(x)+m_{j j}^{2} \lambda_{j}^{2}\right)+\frac{c}{2} u_{j}^{2}=\left\{-\frac{\lambda_{j}+c g_{j}(x)+m_{j j}^{2} \lambda_{j}^{2}}{c}\right\} .
$$

A função é uma quadrática convexa e temos de garantir a positividade pois $u_{j}=z_{j}^{2}$, então tomamos o melhor ponto entre o mínimo global e o zero, definindo

$$
\hat{u}_{j}(x, \lambda)=\max \left\{0,-\frac{\lambda_{j}+m_{j j}^{2} \lambda_{j}^{2}}{c}-g_{j}(x)\right\} .
$$

Por fim, basta tomar $\hat{z}(x, \lambda)=\sqrt{\hat{u}(x, \lambda)}$ coordenada a coordenada e temos finalmente

$$
\min _{x, z, \lambda} w_{c}(x, z, \lambda)=\min _{x, \lambda} w_{c}(x, \hat{z}(x, \lambda), \lambda)
$$

Portanto as variáveis artificiais podem ser removidas da minimização, retornando a um problema só em $x$ e $\lambda$. Como $\lambda$ aparece na definição de $z$, não é mais trivial conseguir uma $M(x)$ que também isole a minimização em $\lambda$ e chegue novamente a um problema só em $x$. Não conhecemos nenhuma penalidade exata que consiga isolar também o $\lambda$.

\subsubsection{Uma penalidade especial}

Como vimos anteriormente, é possível construir penalidades exatas diferenciáveis para problemas de otimização restrita usando um lagrangiano aumentado e uma função que estime o multiplicador a partir do ponto primal. Uma estimativa natural para problemas de desigualdade foi sugerida por Glad e Polak, por meio da solução, por mínimos quadrados, das equações que envolvem os multiplicadores nas condições KKT $\operatorname{com} x$ fixo

$$
\min _{\lambda \in \mathbb{R}^{m}} \phi_{\gamma}(\lambda ; x) \stackrel{\text { def }}{=}\left\|\nabla_{x} L(x, \lambda)\right\|^{2}+\gamma^{2}\|G(x) \lambda\|^{2},
$$


em que $L$ é o lagrangiano usual, $\gamma>0$ é um parâmetro, e $G(x) \in \mathbb{R}^{m \times m}$ é a matriz diagonal $G_{i i}=g_{i}(x)$. O primeiro termo empurra o multiplicador de tal forma a tornar o ponto primal um ponto crítico do lagrangiano. O segundo termo tenta garantir as condições de complementaridade entre os multiplicadores e as restrições. Note que as restrições de desigualdade presentes nas condições KKT não desempenham nenhum papel em (2.12). Sua validade será garantida por uma penalização da inviabilidade do ponto primal e pela hipótese que os problemas tratados são regulares.

O problema (2.12) é quadrático em $\lambda$ e é facilmente resolvido se $x$ for um ponto regular, ou seja, se os gradientes das componentes de $g$ são linearmente independentes em $x$. A seguinte proposição organiza os principais resultados da função multiplicador assim definida. Abaixo $\nabla g$ denota a matriz cujas linhas são os gradientes transpostos das componentes de $g: \mathbb{R}^{n} \rightarrow \mathbb{R}^{m}$, ou seja, $\nabla g$ é o jacobiano de $g$. A hipótese de regularidade, que é a exigência de que os gradientes das componentes de $g$ sejam linearmente independentes em todo ponto é comum na literatura e é conhecida como LICQ (Linear Inequality Constraint Qualification). Para manter a notação mais limpa, omitiremos a dependência em $\gamma$ da solução, pois este parâmetro só será importante no momento da implementação.

Proposição 2.10. Seja $x \in \mathbb{R}^{n}$ ponto regular de $g$ e defina a matriz $N(x) \in \mathbb{R}^{m \times m}$ por

$$
N(x) \stackrel{\text { def }}{=} \nabla g(x) \nabla g(x)^{T}+\gamma^{2} G(x)^{2},
$$

$\operatorname{com} \gamma>0$. Então,

1. $N(x)$ x é definida positiva;

2. A solução única $\lambda(x)$ de $(2.12)$ é

$$
\lambda(x)=-N^{-1}(x) \nabla g(x) \nabla f(x)
$$

3. Se $(\bar{x}, \bar{\lambda})$ é um par KKT de (NLP) com $\bar{x}$ regular, $\lambda(\bar{x})=\bar{\lambda}$.

4. Se existe uma vizinhança de $\bar{x}$ tal que a regularidade vale em toda a vizinhança, $\lambda(x)$ é diferenciável e seu Jacobiano é dado por

$$
\nabla \lambda(x)=-N^{-1}(x)\left[\nabla g(x) \nabla_{x x}^{2} L(x, \lambda(x))+\sum_{i=1}^{m} e_{i} \nabla_{x} L(x, \lambda(x))^{T} \nabla^{2} g_{i}(x)+2 \gamma^{2} \Lambda(x) G(x) \nabla g(x)\right]
$$

onde $e_{i}$ é o $i$-ésimo elemento da base canônica de $\mathbb{R}^{m}$ e $\Lambda(x) \in \mathbb{R}^{m \times m}$ é a matriz diagonal $\Lambda(x)_{i i}=\lambda(x)_{i}$. 
Demonstração. Definindo $I_{\neq}=\left\{i \mid g_{i}(x) \neq 0\right\}$ e o complementar $I_{0}$, temos

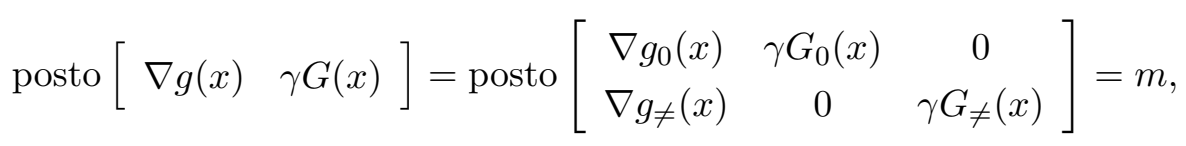

pois pela regularidade de $x$ as primeiras linhas são L.I. entre si e $\gamma G_{\neq}(x)$ é diagonal com todos elementos não nulos. Portanto

$$
N(x)=\left[\begin{array}{ll}
\nabla g(x) & \gamma G(x)
\end{array}\right]\left[\begin{array}{c}
\nabla g(x)^{T} \\
\gamma G(x)
\end{array}\right]
$$

é não-singular e positiva definida.

Temos $\nabla_{x} L(x, \lambda)=\nabla f(x)+\nabla g(x)^{T} \lambda$ e, portanto, (2.12) fica

$$
\min _{\lambda \in \mathbb{R}^{m}}\left\|\nabla f(x)+\nabla g(x)^{T} \lambda\right\|^{2}+\gamma^{2}\|G(x) \lambda\|^{2} .
$$

Podemos resolver esse problema explicitamente. A função a minimizar é

$$
\begin{aligned}
\left(\nabla f(x)+\nabla g(x)^{T} \lambda\right)^{T}\left(\nabla f(x)+\nabla g(x)^{T} \lambda\right)+\gamma^{2}(G(x) \lambda)^{T}(G(x) \lambda) & = \\
\lambda^{T}\left[\nabla g(x) \nabla g(x)^{T}+\gamma^{2} G(x)^{2}\right] \lambda+2 \lambda^{T} \nabla g(x) \nabla f(x)+\nabla f(x)^{T} \nabla f(x) & = \\
\lambda^{T} N(x) \lambda+2 \lambda^{T} \nabla g(x) \nabla f(x)+\nabla f(x)^{T} \nabla f(x), &
\end{aligned}
$$

um problema quadrático em $\lambda$ com hessiana $N(x)$. Pela não-singularidade de $N(x)$, o mínimo existe e é único e encontrado igualando a derivada a zero, ou seja, $N(x) \lambda+\nabla g(x) \nabla f(x)=0$. Portanto $\lambda(x)$ está bem definida.

Se $(\bar{x}, \bar{\lambda})$ é par KKT, temos $\nabla_{x} L(\bar{x}, \bar{\lambda})=0$ e $G(\bar{x}) \bar{\lambda}=0$. Portanto, $\phi_{\gamma}(\bar{\lambda} ; \bar{x})=\left\|\nabla_{x} L(\bar{x}, \bar{\lambda})\right\|^{2}+$ $\gamma^{2}\|G(\bar{x}) \bar{\lambda}\|^{2}=0$. Como $\phi_{\gamma}$ é uma soma do quadrado de normas, ela é sempre positiva. Assim, $\bar{\lambda}$ é uma solução do problema (2.12) para $x=\bar{x}$ e, como o mínimo é único, $\lambda(\bar{x})=\bar{\lambda}$.

Para verificar a fórmula do Jacobiano, inicialmente observamos, da definição de $\lambda(\cdot)$,

$$
\nabla g(x) \nabla g(x)^{T} \lambda(x)+\gamma^{2} G^{2}(x) \lambda(x)=-\nabla g(x) \nabla f(x)
$$

ou seja,

$$
\nabla g(x) \nabla_{x} L(x, \lambda(x))+\gamma^{2} G^{2}(x) \lambda(x)=0 .
$$


Fazendo uma expansão termo a termo da primeira matriz temos

$$
\sum_{j=1}^{m} e_{j} \nabla g_{j}(x)^{T} \nabla_{x} L(x, \lambda(x))+\gamma^{2} G^{2}(x) \lambda(x)=0,
$$

e agora, diferenciando, obtemos

$$
\begin{aligned}
& \sum_{j=1}^{m}\left[e_{j} \nabla_{x} L(x, \lambda(x))^{T} \nabla^{2} g_{j}(x)\right]+\nabla g(x)\left[\nabla_{x x}^{2} L(x, \lambda(x))+\left[\frac{\partial^{2} L(x, \lambda)}{\partial x \partial \lambda}\right]_{\lambda=\lambda(x)} \nabla \lambda(x)\right] \\
+ & \gamma^{2} G^{2}(x) \nabla \lambda(x)+2 \gamma^{2} \Lambda(x) G(x) \nabla g(x)=0 .
\end{aligned}
$$

Lembrando que $\left[\frac{\partial^{2} L(x, \lambda)}{\partial x \partial \lambda}\right]_{\lambda=\lambda(x)}=\nabla g(x)^{T}$, abrindo o segundo termo e isolando $\nabla \lambda(x)$ temos a fórmula do enunciado.

Corolário 2.11. Se vale $L I C Q, \lambda(x)$ é contínua e diferenciável.

Relembrando então a fórmula lagrangiano aumentado clássico para desigualdades,

$$
\begin{aligned}
L_{c}(x, \lambda) & =f(x)+\frac{1}{2 c} \sum_{i=1}^{m}\left(\max \left\{0, \lambda_{i}+c g_{i}(x)\right\}^{2}-\lambda_{i}^{2}\right) \\
& =f(x)+\lambda^{T} g(x)+\frac{c}{2}\|g(x)\|^{2}-\frac{1}{2 c} \sum_{i=1}^{m} \max \left\{0,-\lambda_{i}-c g_{i}(x)\right\}^{2},
\end{aligned}
$$

podemos construir a penalidade $w_{c}(\cdot)$ simplesmente pela substituição direta da estimativa do multiplicador nessa expressão:

$$
w_{c}(x) \stackrel{\text { def }}{=} L_{c}(x, \lambda(x))
$$

Calculando seu gradiente, temos

$$
\begin{aligned}
\nabla w_{c}(x) & =\nabla f(x)+\nabla g(x)^{T} \lambda(x)+\nabla \lambda(x)^{T} g(x)+c \nabla g(x)^{T} g(x) \\
& -\frac{1}{c}\left[-\nabla \lambda(x)^{T}-c \nabla g(x)^{T}\right] \max \{0,-\lambda-c g(x)\} \\
& =\nabla f(x)+\nabla g(x)^{T} \lambda(x)+\left(c \nabla g(x)^{T}+\nabla \lambda(x)^{T}\right) \max \left\{g(x),-\frac{\lambda(x)}{c}\right\} .
\end{aligned}
$$


Por fim, podemos fazer a substituição

$$
y_{c}(x) \stackrel{\text { def }}{=} \max \left\{0,-\frac{\lambda(x)}{c}-g(x)\right\}
$$

coordenada a coordenada, para simplificar a notação e escrever

$$
\begin{array}{r}
w_{c}(x)=f(x)+\lambda(x)^{T} g(x)+\frac{c}{2}\|g(x)\|^{2}-\frac{c}{2}\left\|y_{c}(x)\right\|^{2} \\
\nabla w_{c}(x)=\nabla f(x)+\nabla g(x)^{T} \lambda(x)+\left(c \nabla g(x)^{T}+\nabla \lambda(x)^{T}\right)\left(y_{c}(x)+g(x)\right)
\end{array}
$$

\section{Uma outra visão da penalidade}

Um modo equivalente de chegar à mesma penalidade é fazer como Di Pillo e Grippo e adicionar variáveis extras, transformando as restrições de desigualdade em restrições de igualdade e aplicando o multiplicador no lagrangiano aumentado de igualdades.

O problema fica

$$
\begin{array}{cl}
\min & \hat{f}(x, z)=f(x) \\
\text { s.a } & \hat{h}_{j}(x, z)=g_{j}(x)+z_{j}^{2}=0 j=1 \ldots m .
\end{array}
$$

É importante notar aqui que, como $\nabla \hat{h}_{j}(x, z)=\left(\nabla_{x} g_{j}(x)^{T}, \nabla_{z} e_{j} z_{j}^{2}\right)^{T}$, quando vale a LICQ para as restrições $g(x)$, ela também irá valer para as restrições $\hat{h}(x, z)$.

O lagrangiano aumentado correspondente ao problema acima é

$$
L_{c}(x, z, \lambda)=\hat{f}(x, z)+\lambda^{T}(g(x)+Z z)+\frac{c}{2}\|g(x)+Z z\|^{2},
$$

e mesmo antes de substituir a estimativa do multiplicador podemos observar que, quando formos minimizar $L_{c}$, a minimização em $z$ pode ser separada, pois $L_{c}$ é biquadrática convexa em $z$. Minimizando com relação a $z_{i}$ encontramos o mínimo em $z_{i}^{2}=\max \left\{0,-g_{j}(x)-\frac{1}{c} \lambda_{j}\right\}$. Substituindo $\lambda(x)$ e $z_{c}(x)_{j}^{2}=\max \left\{0,-g_{j}(x)-\frac{1}{c} \lambda(x)_{j}\right\}$ no lagrangiano, chegamos a

$$
\hat{L}_{c}(x)=f(x)+\lambda(x)^{T}\left(g(x)+Z_{c}(x) z_{c}(x)\right)+\frac{c}{2}\left\|g(x)+Z_{c}(x) z_{c}(x)\right\|^{2} .
$$


Se agora abrirmos o último termo, teremos

$$
\begin{aligned}
\hat{L}_{c}(x) \stackrel{\text { def }}{=} & f(x)+\lambda(x)^{T} g(x)+\frac{c}{2}\|g(x)\|^{2}+\frac{c}{2}\left\|Z_{c}(x) z_{c}(x)\right\|^{2} \\
& +\lambda(x)^{T} Z_{c}(x) z_{c}(x)+c g(x)^{T} Z_{c}(x) z_{c}(x) \\
= & f(x)+\lambda(x)^{T} g(x)+\frac{c}{2}\|g(x)\|^{2}+\frac{c}{2}\left\|Z_{c}(x) z_{c}(x)\right\|^{2} \\
+ & +(\lambda(x)+c g(x))^{T} Z_{c}(x) z_{c}(x) .
\end{aligned}
$$

Notamos ainda que, se $\left(\lambda_{i}(x)+c g_{i}(x)\right) \geq 0, z_{c}(x)_{i}^{2}=0$ e o produto desses termos se anula. Já se $\left(\lambda_{i}(x)+c g_{i}(x)\right)<0$, da definição vem $\left(\lambda_{i}(x)+c g_{i}(x)\right)=-c z_{c}(x)_{i}^{2}$ e portanto podemos reescrever a última parcela toda como $-c\left(Z_{c}(x) z_{c}(x)\right)^{T} Z_{c}(x) z_{c}(x)$, recuperando

$$
\hat{L}_{c}(x)=f(x)+\lambda(x)^{T} g(x)+\frac{c}{2}\|g(x)\|^{2}-\frac{c}{2}\left\|Z_{c}(x) z_{c}(x)\right\|^{2} .
$$

Reparando que $z_{c}(x)_{j}^{2}$ é igual o $y_{c}(x)_{j}$ definido anteriormente, temos exatamente $(2.15)$ e $\hat{L}_{c}=w_{c}$. Na prática, o que fizemos foi provar a equivalência dos lagrangianos aumentados para igualdades e desigualdades quando eles estão sendo minimizados, pois em nenhum lugar a escolha específica do multiplicador foi usada. Vamos reescrever $w_{c}$ nessa forma, pois essa variante será interessante em algumas deduções:

$$
w_{c}(x)=f(x)+\lambda(x)^{T}\left(g(x)+y_{c}(x)\right)+\frac{c}{2}\left\|g(x)+y_{c}(x)\right\|^{2} .
$$

\section{Propriedades Interessantes}

Vamos elencar aqui alguns resultados de exatidão dessa penalidade. Começaremos com alguns resultados que falam somente de pontos críticos.

Proposição 2.12. Se $(\bar{x}, \bar{\lambda})$ é KKT para o problema original, para todo $c>0, \nabla w_{c}(\bar{x})=0$ e $w_{c}(\bar{x})=f(\bar{x})$.

Demonstração. A condição KKT e a proposição 2.10 nos dá $\nabla_{x} L(\bar{x}, \lambda(\bar{x}))=0$. Também das condições KKT, $g(x)+y_{c}(x)=0$. Substituindo nas expressões de $w_{c}(2.17)$ e de $\nabla w_{c}(2.16)$ temos a tese.

Queremos agora provar o resultado no outro sentido, ou seja, que pontos críticos de $w$ são pontos KKT. Para isso, precisaremos de dois lemas cujas provas são muito semelhantes àquelas das proposições 3.5 e 3.4, que veremos mais adiante no contexto de penalidades para desigualdades variacionais. Por esse motivo, omitimos as demonstrações. 
Lema 2.13. Se $\hat{x}$ é viável, então existem $c_{\hat{x}}$ e $\sigma_{\hat{x}}$ tais que se $c_{\grave{Z}} c_{\hat{x}}$, para todo $x$ com $\|x-\hat{x}\|<$ $\sigma_{\hat{x}}$ a matriz

$$
K(x ; c)=\left[\nabla g(x) \nabla g(x)^{T}-\gamma^{2} G(x)^{2} Y_{c}(x)+\frac{1}{2 c}\left(\nabla g(x) \nabla \lambda(x)-\gamma^{2} G(x) \Lambda(x)\right)\right]
$$

é não-singular. Além disso se $\nabla w_{c}(x)=0, g(x)+y_{c}(x)=0$.

Lema 2.14. Se vale LICQ e $\nabla w_{c^{k}}\left(x^{k}\right)=0 \operatorname{com} c^{k} \rightarrow \infty$ e $x^{k} \rightarrow \bar{x}$, então $\bar{x}$ é viável.

Como discutido anteriormente, a equivalência contrária à proposição anterior só poderá ser obtida se nos restringirmos a um compacto.

Teorema 2.15. Seja $\mathcal{D}$ um compacto. Se vale $L I C Q$, então existe $\bar{c}$ tal que para todo $c>\bar{c}$, se $\nabla w_{c}(\bar{x})=0$ com $\bar{x} \in \mathcal{D}$, então $(\bar{x}, \lambda(\bar{x}))$ é par KKT para o problema original.

Demonstração. Suponha por absurdo que a tese seja falsa e não exista tal $\bar{c}$ finito. Portanto, podemos tomar seqüências $\left\{x^{k}\right\} \subset \mathcal{D}$ e $\left\{c^{k}\right\}$ tais que $\nabla w_{c^{k}}\left(x^{k}\right)=0, c^{k} \rightarrow \infty$ e o par $\left(x^{k}, \lambda\left(x^{k}\right)\right)$ não é KKT para nenhum $k$.

Pela compacidade de $\mathcal{D}$, podemos tomar uma subseqüência de $x^{k}$, que sem perda de generalidade chamaremos também de $x^{k}$, que converge para um ponto $\hat{x} \in \mathcal{D}$. Caímos no caso do lema 2.14 acima, e $\hat{x}$ é portanto viável. Pelo lema 2.13 há uma vizinhança $V$ de $\hat{x}$ e um limiar $c_{\hat{x}}$ tais que, se $x^{k} \in V$ e $c^{k}>c_{\hat{x}}, K\left(x^{k}, c^{k}\right)$ como definido no mesmo lema é não-singular e $g\left(x^{k}\right)+y_{c^{k}}\left(x^{k}\right)=0$. Pela convergência, $x^{k} \in V$ para $k$ maior que um certo $k_{1}$ e $c^{k}>c_{\hat{x}}$ para $k>k_{2}$.

Tomando o maior dos dois, $k_{0}=\max \left\{k_{1}, k_{2}\right\}$, as hipóteses acima são válidas para todo $k$ maior que $k_{0}$. Como $y_{c}$ é, por definição, maior que zero, isso nos dá $g\left(x^{k}\right) \leq 0$.

Substituindo $g\left(x^{k}\right)+y_{c^{k}}\left(x^{k}\right)=0$ em (2.16), vem direto que $\nabla w_{c^{k}}\left(x^{k}\right)=0$ implica em $\nabla_{x} L\left(x^{k}, \lambda\left(x^{k}\right)\right)=0$. Multiplicando por $\nabla g$ temos

$$
\begin{aligned}
\nabla g\left(x^{k}\right) \nabla_{x} L\left(x^{k}, \lambda\left(x^{k}\right)\right) & =\nabla g\left(x^{k}\right) \nabla f\left(x^{k}\right)+\nabla g\left(x^{k}\right) \nabla g\left(x^{k}\right)^{T} \lambda\left(x^{k}\right) \\
& =-N\left(x^{k}\right) \lambda\left(x^{k}\right)+\nabla g\left(x^{k}\right) \nabla g\left(x^{k}\right)^{T} \lambda\left(x^{k}\right) \\
& =-\gamma^{2} G^{2}\left(x^{k}\right) \lambda\left(x^{k}\right) .
\end{aligned}
$$

O que nós dá a condição de complementaridade $G\left(x^{k}\right) \lambda\left(x^{k}\right)=0$. Se $g_{i}\left(x^{k}\right)=0$, Temos, pela definição de $y_{c}$, e de $y_{c}\left(x^{k}\right)_{i}=0$ que $\lambda_{i}\left(x^{k}\right) \geq 0$. 
Teríamos então que $\left(x^{k}, \lambda\left(x^{k}\right)\right)$ é KKT para todo $k>k_{0}$, absurdo. Portanto, $\exists \bar{c}$ finito e tal que $\forall c>\bar{c}, \nabla w_{c}(\bar{x})=0$ dá que $(\bar{x}, \lambda(\bar{x}))$ é par KKT.

Podemos passar agora a resultados mais específicos, relacionando diretamente os mínimos locais e globais de cada um dos problemas.

\section{Equivalência de Mínimos}

Seja $\mathcal{G}_{\mathcal{P}}$ o conjunto dos mínimos globais do problema original e $\mathcal{G}_{w}(c)$ o dos mínimos globais de $w_{c}$, sempre em um compacto $\mathcal{D}$. Temos o seguinte resultado global:

Teorema 2.16. Seja $\mathcal{D}$ um compacto em que vale $L I C Q$. Se $\emptyset \neq \mathcal{G}_{\mathcal{P}} \subset \operatorname{int} \mathcal{D}$, existe $\bar{c}$ tal que se $c>\bar{c}, \mathcal{G}_{w}(c)=\mathcal{G}_{\mathcal{P}}$.

Demonstração. Vamos começar provando que toda solução global de $w_{c}$ para $c$ suficientemente grande é também solução global do problema original.

Suponha, por absurdo, que exista seqüência $x^{k} \in \mathcal{G}_{w}\left(c^{k}\right) \operatorname{com} x^{k} \notin \mathcal{G}_{P}$ e $c^{k} \rightarrow \infty$. Estamos na mesma situação da seqüência criada na prova do teorema anterior e assim existe $k_{0}$ tal que $g\left(x^{k}\right)+y_{c^{k}}\left(x^{k}\right)=0$ para $k>k_{0}$, o que substituindo na fórmula de $w_{c},(2.17)$, nos dá $w_{c^{k}}\left(x^{k}\right)=f\left(x^{k}\right)$. Se vale LICQ e $\bar{x} \in \mathcal{G}_{\mathcal{P}} \subset \operatorname{int} \mathcal{D}$, existe multiplicador $\bar{\lambda}$ associado formando par KKT e da proposição 2.12 vem $f(\bar{x})=w_{c^{k}}(\bar{x})$. Juntando esses dois resultados e lembrando que $x^{k}$ minimiza $w_{c^{k}}$ temos

$$
f(\bar{x})=w_{c^{k}}(\bar{x}) \geq w_{c^{k}}\left(x^{k}\right)=f\left(x^{k}\right) .
$$

Mas isso, porém, contraria $x^{k} \notin \mathcal{G}_{\mathcal{P}}$, absurdo. Portanto $\mathcal{G}_{w}(c) \subset \mathcal{G}_{P}$ para todo $c$ acima de um limiar $\bar{c}$. Vejamos agora que para esse limiar também vale que $\mathcal{G}_{\mathcal{P}} \subset \mathcal{G}_{w}(c)$.

Tome $\bar{x} \in \mathcal{G}_{\mathcal{P}} \subset \operatorname{int} \mathcal{D}$, mais uma vez usando a proposição 2.12 , sabemos que $f(\bar{x})=w_{c}(\bar{x})$. Agora, tome $\tilde{x} \in \mathcal{G}_{w}(c) \subset \mathcal{G}_{\mathcal{P}}$, que sempre existe pois $D$ é compacto. Como $\tilde{x} \in \mathcal{G}_{\mathcal{P}}, \tilde{x}$ é viável e é também ponto KKT e podemos usar a proposição 2.12 para ver que $w_{c}(\tilde{x})=f(\tilde{x})$. Logo $w_{c}(\tilde{x})=f(\tilde{x}) \geq f(\bar{x})=w_{c}(\bar{x}) \geq w_{c}(\tilde{x})$, de onde concluímos que $\bar{x}$ também minimiza $w_{c}$ em $D$. Portanto segue que vale o outro lado da inclusão e $\mathcal{G}_{w}(c)=\mathcal{G}_{\mathcal{P}}$.

Casos especiais desse teorema incluem a situação em que o conjunto viável original fica todo contido num compacto ou mesmo o caso em que há um único mínimo global, por exemplo 
quando $f$ for estritamente convexa. É interessante também ver que esse resultado nos fornece ferramentas para obter resultados locais.

Corolário 2.17. Se $\bar{x}$ é mínimo local do problema original tal que existe uma bola $B(\bar{x})$ com $\bar{x} \in \operatorname{int} B(\bar{x})$ e tal que $\bar{x}$ seja o mínimo global dentro dessa bola, então existe $\bar{c}$ tal que se $c>\bar{c}$, $\bar{x}$ é mínimo local de $w_{c}$. Se $\bar{x}$ é mínimo local isolado, então existe $\bar{c}$ tal que se $c>\bar{c}, \bar{x}$ é minimo local isolado de $w_{c}$.

A seguir, apresentamos o resultado inverso, com condições que garantem que a minimização irrestrita da penalidade exata $w_{c}$ pode ser usada para recuperar soluções do problema de minimização original. Esse tipo de resultado nos garante então que podemos usar penalidades exatas para desenvolver algoritmos para a solução de problemas de minimização.

Teorema 2.18. Seja $\mathcal{D}$ um compacto em que vale $L I C Q$. Existe $\bar{c}>0$ tal que, se $c>\bar{c}$ e $\bar{x}$ é mínimo local irrestrito de $w_{c}$ e $\bar{x} \in \mathcal{D}$, então $\bar{x}$ é mínimo local do problema original e $\lambda(\bar{x})$ é o multiplicador associado.

Demonstração. Pelo teorema 2.15 temos que para $c$ acima de um certo $\bar{c}$, um mínimo local irrestrito de $w_{c}$ junto com o respectivo multiplicador $(\bar{x}, \lambda(\bar{x}))$ será par KKT. Em particular, $\bar{x}$ é viável. Nessa situação, pela proposição 2.12 ,

$$
w_{c}(\bar{x})=f(\bar{x})
$$

E a definição de mínimo local diz que $f(\bar{x}) \leq w_{c}(x)$ para $x$ em uma vizinhança $V$ de $\bar{x}$.

Suponha agora que $x$ é viável. Se $y_{c}(x)_{i}=0$, vem que $g_{i}(x)+\frac{\lambda_{i}(x)}{c} \geq 0$ e conseqüentemente $c g_{i}(x)^{2}+\lambda_{i}(x) g_{i}(x) \leq 0$. Por outro lado, se $y_{c}(x)_{i}>0, g_{i}(x)+y_{i}(x)=-\frac{\lambda_{i}(x)}{c}$ e portanto

$$
\lambda_{i}(x)\left(g_{i}(x)+y_{c}(x)_{i}\right)+\frac{c}{2}\left(g_{i}(x)+y_{c}(x)_{i}\right)^{2}=-\frac{\left(\lambda_{i}(x)\right)^{2}}{2 c} \leq 0 .
$$

Substituindo essa desigualdade na fórmula de $w_{c},(2.17)$, temos em todos os casos que, para pontos viáveis, $w_{c}(x) \leq f(x)$ e portanto temos

$$
f(\bar{x}) \leq w_{c}(x) \leq f(x)
$$

em $V$. Portanto $\bar{x}$ é mínimo local do problema original.

É importante notar que no contexto de desigualdades variacionais não há como obtermos resultados semelhantes a estes pois não há valores funcionais que possam ser comparados entre 
a penalidade e o problema original. Teremos de achar outra forma de garantir que os pontos KKT são de fato soluções. 


\section{Capítulo 3}

\section{Penalidades Exatas para Desigualdades Variacionais}

Nosso objetivo é estender a definição de $w_{c}$ para o contexto das desigualdades variacionais. Vamos rapidamente revisar alguns conceitos sobre elas na seção 3.1. Para um estudo mais detalhado, remetemos o leitor a algumas referências $[42,21,20]$. O restante do capítulo consiste no núcleo de nosso trabalho e os resultados contidos a partir da seção 3.2 são fruto de nossa pesquisa e são, em geral, originais, a menos do teorema da subseção 3.2.2. A seção 3.2 apresenta a definição de uma penalidade para problemas de desigualdade variacional e prova resultados de exatidão. A seção 3.3 apresenta resultados mais finos para um caso particular e importante de desigualdades variacionais: complementaridade não-linear.

\subsection{Desigualdades Variacionais}

A solução $\bar{x}$ de um problema de otimização irrestrita na forma

$$
\min _{x} f(x)
$$

em que $f$ é diferenciável é necessariamente um ponto tal que $\nabla f(\bar{x})=0$. Se a função é convexa, essa condição também é suficiente para que se encontre uma solução, ou seja, podemos encontrar uma solução do problema inicial se resolvermos o problema de encontrar um zero do seu gradiente. No caso de um problema com restrições

$$
\min _{x \in C} f(x)
$$


temos que, teoricamente, tudo se passa como se resolvêssemos um problema irrestrito para a função $f+I_{C}$, em que $I_{C}$ é a função indicadora do conjunto restrição, valendo 0 nele e $+\infty$ fora. Essa função é convexa se e somente se $C$ for um conjunto convexo, em particular se $C=\{x-g(x) \leq 0\}$ com as coordenadas de $g$ convexas. Se $f$ é convexa, a condição necessária e suficiente para que $\bar{x}$ seja solução é que $\bar{x}$ seja um zero do operador subdiferencial $\partial\left(f+I_{C}\right)$. Se $f$ é diferenciável e dom $f$ é todo o espaço ${ }^{1}$, temos $\partial\left(f+I_{C}\right)(x)=\nabla f(x)+\partial I_{C}(x)=$ $\nabla f(x)+N_{C}(x)$ em que $N_{C}$ é o cone normal ao conjunto $C$. Usando a definição de cone normal, podemos simplesmente reescrever o problema $0 \in \nabla f(x)+N_{C}(x)$ como o de encontrar $\bar{x} \in C$ tal que $N_{C}(\bar{x}) \ni-\nabla f(\bar{x})$, ou seja,

$$
\langle\nabla f(\bar{x}), y-\bar{x}\rangle \geq 0, \forall y \in C
$$

E é fácil reconhecer aí as condições geométricas de primeira ordem de otimalidade de PNL $[34,35]$, que valem mesmo no caso em que $f$ é não convexa.

O problema generalizado de desigualdade variacional é exatamente resolver (3.1), substituindo $\nabla f$ por um operador genérico $F$, possivelmente ponto-conjunto. Esse problema tem uma série de aplicações em áreas como EDPs, engenharia, física e economia [11, 21].

Nesse trabalho exigiremos que a função presente na desigualdade $F: \mathbb{R}^{n} \rightarrow \mathbb{R}^{n}$ seja contínua e a restrição será $\mathcal{F} \subset \mathbb{R}^{n}$, um conjunto fechado não-vazio que será descrito na forma $\mathcal{F}=\{x-g(x) \leq 0\}$ com $g_{i}$ convexas. O nosso problema de desigualdade variacional é encontrar $x \in \mathcal{F}$ tal que

$$
\forall y \in \mathcal{F}, \quad F(x)^{T}(y-x) \geq 0
$$

Tais hipóteses sobre $F$ e $\mathcal{F}$ ainda abrangem muitas aplicações e são as mesmas tomadas, por exemplo, em [21].

\subsubsection{Métodos para resolver Desigualdades Variacionais}

Assim como nossa definição da desigualdade variacional foi inspirada numa generalização das condições de primeira ordem do problema de programação não-linear em que substituímos o diferencial de uma função por um operador genérico, o mesmo tipo de substituição pode ser usada para adaptar métodos de otimização para resolver problemas de desigualdades variacionais. Mencionaremos rapidamente alguns desenvolvimentos possíveis de métodos, sem apresentar todos os detalhes. O leitor interessado encontrará bom material de estudo nas

\footnotetext{
${ }^{1} \mathrm{Na}$ verdade basta que int $\operatorname{dom} f \supset C$.
} 
referências citadas.

Entre os métodos mais estudados estão os métodos de multiplicadores. No caso de programação não-linear esses métodos estão intimamente ligados aos métodos proximais, algoritmos em que uma forma generalizada de função distância $D: \mathbb{R}^{n} \times \mathbb{R}^{n} \rightarrow \overline{\mathbb{R}}$ é usada para se resolver iterativamente o problema

$$
x^{k}=\underset{g(x) \leq 0}{\arg \min } f(x)+\alpha_{k} D\left(x ; x^{k-1}\right) .
$$

O problema acima pode ser bem mais fácil de resolver do que a minimização não regularizada por $D$, e para $f$ convexa existem provas da convergência desse método a uma solução do problema original para uma série de possíveis restrições $g$ e escolhas de distâncias generalizadas d. A literatura do assunto é muito extensa e remetemos o leitor inicialmente a [40, 44, 43, 41].

Grande parte do interesse que temos aqui nos métodos proximais está na relação com os métodos de multiplicadores. Um dos resultados mais importantes é que a aplicação do método proximal ao problema dual irá gerar, a cada passo, soluções $\bar{\lambda}^{k}$ que satisfazem a equação

$$
\bar{\lambda}^{k}=\nabla P\left(g\left(\bar{x}^{k}\right) ; \bar{\lambda}^{k-1}\right)
$$

em que $\bar{x}^{k}$ é um ponto primal calculado por um método de multiplicadores. $P$ é a função convexa conjugada a $D\left(\cdot ; \lambda^{k-1}\right)+I_{\mathbb{R}_{+}^{n}}(\cdot)$ e é também é a função que faz o papel da penalidade no método de multiplicadores, definindo o problema a ser resolvido a cada passo:

$$
x^{k}=\arg \min f(x)+c_{k} P\left(g(x) ; \bar{\lambda}^{k-1}\right)
$$

com posterior atualização de $\bar{\lambda}^{k-1} \operatorname{com}(3.3)$. O caso em que $D\left(\lambda_{1}, \lambda_{2}\right)=\frac{1}{2}\left\|\lambda_{1}-\lambda_{2}\right\|^{2}$ recupera o clássico método de Rockafellar [40] e outras escolhas de $D$ bem como uma análise mais aprofundada desses resultados podem ser observadas em [44].

A grande importância aqui está em notar o papel fundamental que tem a construção de um problema dual. As prova da convergência do método proximal e da equivalência das soluções desse com as do método de multiplicadores é que vão trazer a convergência do método de multiplicadores. Ao se tentar, portanto, desenvolver esse tipo de método para desigualdades variacionais, torna-se necessário criar uma formulação dual para o problema, escolher uma classe de funções $D$, construir um método proximal que convirja e provar a equivalência das soluções a cada passo com as do método original.

Um primeiro problema é então o de construir um problema dual a uma desigualdade 
variacional. Retomando nosso problema original

$$
0 \in F(x)+N_{\mathcal{F}}(x)
$$

uma primeira tentativa de criar um problema dual vem de Mosco, ainda em 1972 [30]. No seu caso o problema tinha a forma $0 \in F(x)+\partial f_{0}(x)$ em que $\partial f_{0}$ é o subdiferencial de uma função convexa $f_{0}$. Sua formulação dual era

$$
0 \in-F^{-1}(y)+\partial f_{0}^{*}(y)
$$

em que $f_{0}^{*}$ é a função convexa conjugada a $f_{0}$ e a inversa de $F$ é tomada no contexto de operadores ponto-conjunto $F^{-1}(y)=\{x-F(x)=y\}$.

Uma variação mais geral é a de Attouch e Théra [1], já em 1996, considerando somas mais gerais

$$
\zeta \in T(x)+S(x)
$$

com dual

$$
0 \in T^{-1}(y)-S^{-1}(\zeta-y)
$$

que é inspirada na dualidade de Fenchel-Rockafellar-Moureau [39]. Essa formulação pode ser construída notando que $x$ é uma solução do problema primal se e somente se existe $y$ tal que

$$
y \in T(x) \text { e } \zeta-y \in S(x)
$$

e por outro lado, dado um $y$, a existência de um $x$ que resolva as equações acima é equivalente a $y$ resolver (3.4), em que as inversas são tomadas também como operadores ponto-conjunto.

No caso da desigualdade variacional o problema dual fica

$$
0 \in F^{-1}(y)-\left(N_{\mathcal{F}}\right)^{-1}(-y)
$$

Uma das características que aparecem aqui é a ausência de uma clara formulação lagrangiana. Uma forma primal-dual pode ser necessária para se desenvolver alguns tipos de algoritmos, mas uma formulação que só usa a construção acima já é possível.

Em 1999, usando exatamente esse tipo de substituição, Eckstein e Ferris fizeram uma extensão de métodos de multiplicadores para problemas de complementaridade não-linear mistos [18], desigualdades variacionais em que $\mathcal{F}$ é uma caixa. De fato, para o problema de complementaridade em que $\mathcal{F}=\mathbb{R}_{+}^{n}$, temos que $(-I) \circ\left(N_{\mathbb{R}_{+}^{n}}\right)^{-1} \circ(-I)=N_{\mathbb{R}_{+}^{n}}$ e a formulação 
dual fica

$$
0 \in F^{-1}(y)+N_{\mathbb{R}_{+}^{n}}(y)
$$

que é uma desigualdade variacional com mesmo conjunto viável, possivelmente com um operador ponto-conjunto como objetivo.

Um método proximal (3.2) aplicado ao problema convexo $\min _{g(x) \leq 0} f(x)$ é equivalente a resolver iterativamente

$$
0 \in \nabla f\left(x^{k+1}\right)+N_{C}\left(x^{k+1}\right)+\alpha_{k} \nabla D\left(x^{k+1} ; x^{k}\right),
$$

em que $C=\{x-g(x) \leq 0\}$. Substituindo essa forma no problema original (PDV), teremos a forma

$$
0 \in F\left(x^{k+1}\right)+N_{\mathcal{F}}\left(x^{k+1}\right)+\alpha_{k} \nabla D\left(x^{k+1} ; x^{k}\right)
$$

em que $D$ é uma distância generalizada como em programação não-linear. Uma aplicação desse tipo de método à formulação dual (3.6) nos traz

$$
0 \in F^{-1}\left(y^{k+1}\right)+N_{\mathbb{R}_{+}^{n}}\left(y^{k+1}\right)+\alpha_{k} \nabla D\left(y^{k+1} ; y^{k}\right)
$$

e aplicando a transformação de dualidade (3.4) sobre essa equação, com $T=F^{-1}$ e $S=$ $N_{\mathbb{R}_{+}^{n}}+\alpha_{k} \nabla D\left(\cdot ; y^{k}\right)=\alpha_{k}\left(N_{\mathbb{R}_{+}^{n}}+\nabla D\left(\cdot ; y^{k}\right)\right)$ teremos o problema equivalente

$$
0 \in F\left(x^{k+1}\right)-\left(N_{\mathbb{R}_{+}^{n}}+\nabla D\left(\cdot ; y^{k}\right)\right)^{-1}\left(-\frac{1}{\alpha_{k}} x^{k+1}\right) .
$$

Com algumas condições sobre $D$, como convexidade estrita com relação a primeira coordenada, podemos separar a função univalorada

$$
\nabla P\left(\cdot, y^{k}\right) \stackrel{\text { def }}{=}\left(N_{\mathbb{R}_{+}^{n}}+\nabla D\left(\cdot ; y^{k}\right)\right)^{-1}
$$

em que nos valemos de um certo abuso de notação, e escrever como problema equivalente ao método proximal as recursões

$$
\begin{aligned}
& 0=F\left(x^{k+1}\right)-\nabla P\left(-\frac{1}{\alpha_{k}} x^{k+1} ; y^{k}\right) \\
& y^{k+1}=\nabla P\left(-\frac{1}{\alpha_{k}} x^{k+1} ; y^{k}\right)
\end{aligned}
$$

que é um tipo de método de multiplicadores para desigualdades variacionais.

Estratégias como essa já foram desenvolvidas e transformadas em algoritmos para classes ainda maiores de restrições do que os problemas de complementaridade. Eckstein e Ferris [18] 
e depois Eckstein e Silva [45] aplicaram métodos como esses a restrições de caixas genéricas ao conseguir uma boa representação para $N_{\mathcal{F}}^{-1}$ e usando funções de Bregman como a distância $D$. Pouco tempo depois Auslender e Teboulle aproveitando-se das idéias de [6, 3] propuseram uma diferente forma de dualidade e conseguiram uma extensão do conceito de lagrangiano aumentado para problemas de desigualdade variacional, assim como a definição de um problema lagrangiano, ou primal-dual $[4,5]$. Esses trabalhos permitiram que eles obtivessem resultados de convergência para $\mathcal{F}$ determinados por desigualdades convexas genéricas, quando as distâncias $D$ estão na classe das $\Phi$-divergências. Usando regularizações duplas, que combinam esses tipos de distâncias especiais e a distância quadrática clássica, Eckstein e Silva conseguem resultados ainda mais gerais [45]. Todas a provas exigem de uma forma ou outra a monotonicidade da função $F$, embora bons resultados práticos tenham sido obtidos com a aplicação dos algoritmos mesmo a problemas não monótonos [18, 45].

Os melhores resultados de dualidade de Auslender e Teboulle estão relacionados a uma rica teoria de dualidade para equações generalizadas desenvolvida por Pennanen [35, 34]. Essa teoria ataca o problema de forma bastante abstrata e permite uma construção completa de problemas primal, dual e primal-dual e é a mais geral de todas. Ela assemelha-se a teoria de dualidade conjugada de programação não-linear e permite uma série de associações entre os resultados nesse contexto e o das equações generalizadas, em particular as desigualdades variacionais. Um estudo nessa linha foge do escopo desse trabalho, mas é importante notar que métodos podem ser obtidos mesmo com substituições simples de $\nabla f$ pela função $F$, como fizemos acima.

\subsection{Estendendo Penalidades Exatas para Desigualdades Vari- acionais}

Seguindo esse avanços, uma forma natural de estender a noção de penalidade exata para (PDV) é usar o gradiente de $w_{c}$, substituindo onde necessário o gradiente $\nabla f$ pelo operador $F$. Se tudo correr bem, teríamos só de resolver a equação $\nabla w_{c}(x)=0$ para encontrar a solução da desigualdade variacional.

Vamos retomar a fórmula de $\nabla w_{c}$ de $(2.14)$ :

$$
\nabla w_{c}=\nabla f(x)+\nabla g(x)^{T} \lambda(x)+\left(c \nabla g(x)^{T}+\nabla \lambda(x)^{T}\right) \max \left\{g(x),-\frac{\lambda(x)}{c}\right\}
$$

em que 
$\nabla \lambda(x)=-N^{-1}(x)\left[\nabla g(x) \nabla_{x x}^{2} L(x, \lambda(x))+\sum_{i=1}^{m} e_{i} \nabla_{x} L(x, \lambda(x))^{T} \nabla^{2} g_{i}(x)+2 \gamma^{2} \Lambda(x) G(x) \nabla g(x)\right]$,

e $N(x)=\nabla g(x) \nabla g(x)^{T}+\gamma^{2} G(x)^{2}$. Para poder usar exatamente estas fórmulas no caso de desigualdades variacionais, teremos de substituir $\nabla f$ por $F$ em $\nabla w_{c}$ e também nos lagrangianos. Escreveremos, com um abuso de notação, $\nabla_{x} L(x, \lambda(x)) \stackrel{\text { def }}{=} F(x)+\lambda(x)^{T} \nabla g(x) \mathrm{e}$ $\nabla_{x x}^{2} L(x, \lambda(x)) \stackrel{\text { def }}{=} \nabla F(x)+\lambda(x)^{T} \sum_{i=1}^{m} e_{i} \nabla^{2} g_{i}(x)$, em que $\nabla F$ entrou no lugar de $\nabla^{2} f$, a hessiana de $f$.

Como fica claro, esse gradiente de $w_{c}$ seria muito complicado e trabalhoso de se calcular, pois nele aparece o Jacobiano de $\lambda(\cdot)$, que depende da Hessiana das restrições e do gradiente do operador $F$, que podem ser difíceis de obter. Um método para resolver $\nabla w_{c}(x)=0$ necessitaria, provavelmente, de informações de ordem ainda mais alta, o que limitaria bastante as possibilidades de uso desse método para resolver desigualdades variacionais.

Apesar das dificuldades em operar com o jacobiano de $\lambda(\cdot)$ e do custo computacional de calculá-lo em um algoritmo, observando as provas dos resultados para a penalidade em PNL, podemos notar que a fórmula de $\nabla \lambda$ nunca é usada diretamente e, mais, em nenhum resultado o termo que envolve $\nabla \lambda$ nos pareceu essencial para obter a demonstração, sendo muitas vezes assintoticamente desprezível. Por mais que essa constatação seja válida apenas para as provas e não para os resultados algorítmicos da penalidade, uma idéia natural então é simplesmente se livrar desse termo e tentar provar os resultados para uma penalidade nova, que possivelmente terá as mesmas propriedades de convergência e com as vantagens computacionais que a omissão do termo de $\nabla \lambda$ traz.

Ficamos então com

$$
W_{c}(x) \stackrel{\text { def }}{=} F(x)+\nabla g(x)^{T} \lambda(x)+c \nabla g(x)^{T} \max \{g(x),-\lambda(x) / c\} .
$$

De fato, nas provas de nossos resultados, a ausência do termo de $\nabla \lambda$ não trouxe qualquer dificuldade. Antes de passarmos aos resultados propriamente ditos, vejamos uma outra forma de chegar a essa mesma penalidade partindo de uma outra estratégia, com maior respaldo teórico. 


\subsubsection{O Lagrangiano Aumentado de Desigualdades Variacionais}

Para driblar a dificuldade de calcular a penalidade adaptada de PNL, enfrentaremos o problema de outra maneira. Usaremos a mesma estratégia de aplicar uma estimativa do multiplicador ao lagrangiano aumentado, mas partindo do lagrangiano aumentado clássico para desigualdades variacionais, que é o gradiente de $L_{c}(\cdot, \cdot)$ com relação à primeira variável [4].

Colocamos a mesma estimativa do multiplicador usada em PNL, $\lambda(\cdot)$,

$$
\begin{aligned}
\lambda(x) & \stackrel{\text { def }}{=}-N^{-1}(x) \nabla g(x) F(x), \\
W_{c}(x) & \left.\stackrel{\text { def }}{=} \nabla_{x} L_{c}(x, \lambda)\right|_{(x, \lambda(x))} \\
& =F(x)+\nabla g(x)^{T} \lambda(x)+c \nabla g(x)^{T} g(x)+c \nabla g(x)^{T} \max \{0,-\lambda(x) / c-g(x)\} \\
& =F(x)+\nabla g(x)^{T} \lambda(x)+c \nabla g(x)^{T} \max \{g(x),-\lambda(x) / c\} .
\end{aligned}
$$

que é mesma função que obtemos ao remover os termos de $\nabla \lambda$ da penalidade de PNL.

Antes de provar as propriedades de exatidão de $W_{c}$, é importante destacar alguns fatos. Antes de mais nada, por partir da derivada de $L_{c}$ somente com relação a $x, W_{c}$ não é o gradiente de $w_{c}$ e portanto seus zeros não estão claramente relacionados a soluções de um problema de otimização irrestrita. Nesse sentido essa abordagem para criação de uma penalidade exata difere das usualmente encontradas na literatura. A sua grande vantagem é não depender das segundas derivadas de nenhum dos operadores envolvidos.

Outro detalhe que deve ser destacado são as propriedades de diferenciabilidade de $W_{c}$. A função max na fórmula irá torná-la não-diferenciável. Essa característica é herdada diretamente do lagrangiano aumentado clássico que foi usado para gerá-la. Mesmo assim, ela é semi-suave e seus zeros podem ainda ser encontrados por extensões do método de Newton $[37,38]$. Nesse sentido, $W_{c}$ pode ser visto como uma variação das funções NCP, como a própria função max ou a função de Fischer-Burmeister [22], só que incorporando informação dual por meio das estimativas do multiplicador.

\subsubsection{O sistema KKT de Desigualdades Variacionais}

Nas seções a seguir mostraremos a relação entre os zeros de $W_{c}$ para $c$ suficientemente grande e o sistema KKT associado ao (PDV). 
Definição 3.1. O sistema Karush-Kuhn-Tucker (KKT) associado ao (PDV) é

$$
\begin{aligned}
F(x)+\nabla g(x)^{T} \lambda & =0 \\
g(x) & \leq 0, \\
\lambda & \geq 0, \\
\forall i=1, \ldots, m, \lambda_{i} g_{i}(x) & =0 .
\end{aligned}
$$

(Complementariedade)

Um par $(x, \lambda) \in \mathbb{R}^{n \times m}$ que satisfaça a todas essas equações é chamado de par KKT. A variável primal, $x$ é chamada de ponto KKT.

Precisamos verificar que de fato podemos chamar esse conjunto de equações de um sistema KKT, ou seja, precisamos provar que assim como em otimização, resolver o sistema é equivalente a resolver a desigualdade variacional original. Em PNL, a convexidade da função objetivo e das restrições garante que uma solução do sistema KKT é solução do problema original, e se as restrições satisfizerem uma condição de qualificação como a LICQ ou a condição de Slater, temos que toda solução possui multiplicador associado e é também ponto KKT.

Pela estrutura do lagrangiano aumentado em desigualdades variacionais, basta exigir a convexidade das restrições, sem que tenham de ser feitas hipóteses de monotonicidade sobre $F$.

Teorema 3.2. O sistema KKT acima definido é equivalente ao problema (PDV) quando o conjunto $\mathcal{F}$ for definido por desigualdades convexas e satisfizer uma condição de qualificação.

Demonstração. Considere que $\mathcal{F}=\{x-g(x) \leq 0\}$. Para encontrar a equivalência de soluções, é útil considerar o seguinte problema de minimização em $y$ com parâmetro $\bar{x}$,

$$
\begin{aligned}
& \min _{y} F(\bar{x})^{T}(y-\bar{x}) \\
& \text { s.a } g(y) \leq 0
\end{aligned}
$$


O sistema KKT, ainda em PNL, de $\left(P_{\text {aux }}\right)$ é

$$
\begin{aligned}
F(\bar{x})+\nabla g(y)^{T} \lambda & =0, \\
g(y) & \leq 0, \\
\lambda & \geq 0, \\
\forall i=1, \ldots, m, \lambda_{i} g_{i}(y) & =0 .
\end{aligned}
$$

e basta que as coordenadas de $g$ sejam convexas e que satisfaçam uma condição de qualificação para que esse sistema e $\left(P_{\text {aux }}\right)$ sejam equivalentes, pois a função objetivo é linear.

Mas se $\tilde{x}$ é a solução de um problema de desigualdade variacional, por definição $F(\tilde{x})^{T}(y-\tilde{x}) \geq$ 0 para todo $y \in \mathcal{F}$ e $F(\tilde{x})^{T}(\tilde{x}-\tilde{x})=0$, portanto $\tilde{x}$ é solução de $\left(P_{\text {aux }}\right)$ com parâmetro $\tilde{x}$ e também do seu sistema KKT.

Seja agora um par $(\hat{x}, \hat{\lambda})$ que resolve o sistema KKT proposto para desigualdade variacionais. Ele claramente resolve o sistema $\operatorname{KKT}$ de $\left(P_{\text {aux }}\right)$, $\operatorname{com} \bar{x}=\hat{x}, \lambda=\hat{\lambda}$ e $y=\hat{x}$. Portanto $\hat{x}$ é solução do $\left(P_{\text {aux }}\right)$ com parâmetro $\hat{x}$, ou seja,

$$
\begin{gathered}
g(\hat{x}) \leq 0 \\
\forall y \in \mathcal{F}, \quad F(\hat{x})^{T}(y-\hat{x}) \geq F(\hat{x})^{T}(\hat{x}-\hat{x})=0,
\end{gathered}
$$

que é a condição para resolver a desigualdade variacional.

Concluímos então que qualquer dos dois problemas, (PDV) ou o sistema KKT proposto têm exatamente as mesmas soluções.

\subsubsection{Exatidão da Penalidade}

Vamos apresentar nessa seção os resultados de exatidão de $W_{c}$. Assumiremos daqui em diante que $\mathcal{F}=\{x-g(x) \leq 0\}$ e vale a LICQ em todo $\mathbb{R}^{n}$, de modo que $\lambda(\cdot)$ e portanto $W_{c}(\cdot)$ estão definidas em todo lugar, o que também é uma das condições que garante a equivalência entre a desigualdade variacional e o sistema KKT acima. Começaremos mostrando que essa penalidade tem zeros nas soluções do problema original.

Proposição 3.3. Seja $(x, \lambda)$ um par KKT. Então $\forall c>0,0=W_{c}(x)$. 
Demonstração. A partir da LICQ, a proposição 2.10 garante que $\lambda=\lambda(x)$, temos então

$$
\begin{aligned}
W_{c}(x) & =F(x)+\nabla g(x)^{T} \lambda(x)+c \nabla g(x)^{T} \max \{g(x),-\lambda(x) / c\} \\
& =0+c \nabla g(x)^{T} \max \{g(x),-\lambda(x) / c\}
\end{aligned}
$$

pela condição do zero de KKT. Mas pela condição de viabilidade primal vem que $g(x) \leq 0$, e pela viabilidade dual e da positividade de $c$ vem que $-\frac{\lambda(x)}{c} \leq 0$. Da condição de complementaridade vem que, coordenada a coordenada, sempre um dos dois termos entre $g_{i}(x)$ e $\lambda_{i}(x)$ se anula, ou seja, $\max \{g(x),-\lambda(x) / c\}=0$ e portanto temos que $W_{c}(x)$ se anula.

Seguiremos aqui as mesmas idéias utilizadas em PNL. Vejamos mais algumas propriedades de $W_{c}$.

Proposição 3.4. Sejam $\left\{x^{k}\right\} \subset \mathbb{R}^{n}$ e $\left\{c_{k}\right\} \subset \mathbb{R}_{+}$seqüências tais que $x^{k} \rightarrow \bar{x}, c_{k} \rightarrow+\infty$. Se $0=W_{c_{k}}\left(x^{k}\right)$, temos $\bar{x} \in \mathcal{F}$.

Demonstração. Temos da definição,

$$
0=W_{c_{k}}\left(x^{k}\right)=F\left(x^{k}\right)+\nabla g\left(x^{k}\right)^{T} \lambda\left(x^{k}\right)+c_{k} \nabla g\left(x^{k}\right)^{T} \max \left\{g\left(x^{k}\right),-\lambda\left(x^{k}\right) / c_{k}\right\} .
$$

O corolário 2.11 garante que $\lambda(\cdot)$ é contínuo. Como assumimos desde o início que $F$ é contínua e $g$ é continuamente diferenciável, podemos dividir a equação acima por $c_{k}$ e em seguida tomar os limites, anulando os dois primeiros termos:

$$
0=\sum_{i=1}^{m} \max \left\{g_{i}(\bar{x}), 0\right\} \nabla g_{i}(\bar{x}) .
$$

Pela própria definição da LICQ temos que todas as constantes multiplicando os gradientes das componentes de $g$ devem ser nulas para que uma combinação linear das mesmas se anule. Portanto $\max \left\{g_{i}(\bar{x}), 0\right\}=0$ para todo $i=1, \ldots, m$. Conseqüentemente, $g(\bar{x}) \leq 0$ e $\bar{x}$ é viável.

Proposição 3.5. Seja $\bar{x} \in \mathcal{F}$. Então existem $c_{\bar{x}}, \delta_{\bar{x}}>0$ tais que se valem $\|x-\bar{x}\| \leq \delta_{\bar{x}}, c>c_{\bar{x}}$ e $0 \in W_{c}(x)$, então $(x, \lambda(x))$ é um par KKT do problema (PDV).

Demonstração. Vamos simplificar nossa notação usando

$$
y_{c}(x) \stackrel{\text { def }}{=} \max \left\{0,-\frac{\lambda(x)}{c}-g(x)\right\} .
$$


Mais uma vez, letras maiúsculas serão usadas para representar matrizes diagonais compostas a partir de um vetor. Por exemplo, $Y_{c}(x)$ é a matriz diagonal com as componentes de $y_{c}(x)$ na diagonal.

Da definição temos que se $y_{c}(x)_{i} \neq 0, y_{c}(x)_{i}=-\lambda_{i}(x) / c-g_{i}(x)$ e $\lambda_{i}(x)=-c\left(y_{c}(x)_{i}+g_{i}(x)\right)$. Portanto

$$
Y_{c}(x) \lambda(x)=-c Y_{c}(x)\left(g(x)+y_{c}(x)\right)
$$

Temos então,

$$
\begin{aligned}
\nabla g(x) \nabla_{x} L(x, \lambda(x)) & =\nabla g(x) F(x)+\nabla g(x) \nabla g(x)^{T} \lambda(x) \\
& =-N(x) \lambda(x)+\nabla g(x) \nabla g(x)^{T} \lambda(x) \\
& =-\gamma^{2} G(x)^{2} \lambda(x) \\
& =-\gamma^{2} G(x)\left(G(x)+Y_{c}(x)\right) \lambda(x)+\gamma^{2} G(x) Y_{c}(x) \lambda(x) \\
& =-\gamma^{2} G(x) \Lambda(x)\left(g(x)+y_{c}(x)\right)+\gamma^{2} G(x) Y_{c}(x) \lambda(x)
\end{aligned}
$$

Combinando esse último termo com a equação anterior segue que

$$
\frac{1}{c} \nabla g(x) \nabla_{x} L(x, \lambda(x))=-\gamma^{2} G(x)\left(\frac{1}{c} \Lambda(x)+Y_{c}(x)\right)\left(g(x)+y_{c}(x)\right) .
$$

E, da definição de $W_{c}$, temos

$$
\begin{aligned}
\frac{1}{c} \nabla g(x) W_{c}(x) & =\frac{1}{c} \nabla g(x) \nabla_{x} L(x, \lambda(x))+\nabla g(x) \nabla g(x)^{T}\left(g(x)+y_{c}(x)\right) \\
& =K(x, c)\left(g(x)+y_{c}(x)\right)
\end{aligned}
$$

em que definimos $K(x, c) \stackrel{\text { def }}{=}\left(\nabla g(x) \nabla g(x)^{T}-\gamma^{2} G(x) Y_{c}(x)\right)-\frac{1}{c} \gamma^{2} G(x) \Lambda(x)$.

Como $\bar{x}$ é viável por hipótese, se $x \rightarrow \bar{x}$ e $c \rightarrow+\infty$ então, pela continuidade de $g(\cdot)$ e $\lambda(\cdot)$, vem que $y_{c}(x) \rightarrow-g(\bar{x})$ e portanto, da continuidade $K$ em $x$ e $c$, temos que $K(x, c) \rightarrow N(\bar{x})$. Como $N(\bar{x})$ é não-singular por causa da LICQ, a continuidade garante que existem $c_{\bar{x}}, \delta_{\bar{x}}>0$ tais que se $\|x-\bar{x}\| \leq \delta_{\bar{x}}, c>c_{\bar{x}}$ então $K(x, c)$ também é não-singular.

Sejam $x, c$ nessas condições, ou seja, $\|x-\bar{x}\| \leq \delta_{\bar{x}}, c>c_{\bar{x}}$ e suponha que $0 \in W_{c}(x)$. Então, (3.13) e a não-singularidade de $K(x, c)$ implicam que $g(x)+y_{c}(x)=0$. Retornando este resultado em (3.12) e usando novamente a LICQ temos que $\nabla_{x} L(x, \lambda(x))=0$, a condição 
zero de KKT. Finalmente, $g(x)+y_{c}(x)=0$ é o mesmo que dizer

$$
\begin{gathered}
\max \{g(x),-\lambda(x) / c\}=0 \Longrightarrow \\
g(x) \leq 0, \quad \lambda(x) \geq 0,
\end{gathered}
$$

viabilidades primal e dual. Para terminar, voltando à passagem (3.11) e usando a condição zero já estabelecida, temos a complementaridade, confirmando que $(x, \lambda(x))$ é um par KKT.

Essa prova é muito semelhante à usada por Di Pillo e Grippo em seus artigos para PNL, sem as dificuldades associadas à necessidade de operar sobre as derivadas de $\lambda(x)$.

Podemos unir os dois resultados anteriores para formular o seguinte teorema.

Teorema 3.6. Assuma que vale a $L I C Q$ em todo $\mathbb{R}^{n}$, de modo que $\lambda(\cdot)$ e $W_{c}(\cdot)$ estão definidas em todo lugar. Sejam $\left\{x^{k}\right\} \in \mathbb{R}^{n}$ e $\left\{c_{k}\right\} \in \mathbb{R}$ seqüências tais que $0=W_{c_{k}}\left(x^{k}\right), c_{k} \rightarrow+\infty$, e $\left\{x^{k}\right\}$ é limitado. Então existe um índice finito $K$ tal que $\left(x^{K}, \lambda\left(x^{K}\right)\right)$ é uma solução $K K T$ associada ao (PDV) para todo $k>K$.

Demonstração. Suponha por contradição que podemos extrair uma subseqüência $\left\{x^{k_{j}}\right\}$ de pontos que não são KKT. Como $\left\{x^{k}\right\}$ é limitada, podemos supor sem perda de generalidade que $\left\{x^{k_{j}}\right\}$ converge para um certo $\bar{x} \in \mathbb{R}^{n}$. Usando a proposição 3.4 , concluímos que $\bar{x}$ é viável. Podemos então aplicar a proposição 3.5. Das hipóteses, temos que existe $K$ finito tal que $\left\|x^{k}-\bar{x}\right\| \leq \delta_{\bar{x}}$ e $c_{k}>c_{\bar{x}}$, para todo $k>K$, garantindo que $\left(x_{k}, \lambda\left(x_{k}\right)\right)$ é solução, o que é uma contradição.

Corolário 3.7. Assuma que vale a LICQ em todo $\mathbb{R}^{n}$, então temos $\lambda(\cdot)$ e $W_{c}(\cdot)$ definidas em todo lugar. Suponha que existe $\tilde{c} \in \mathbb{R}$ tal que o conjunto $\left\{x \mid 0 \in W_{c}(x), c>\tilde{c}\right\}$ é limitado. Então existe $\bar{c}>0$ tal que se $0 \in W_{c}(x)$ e $c>\bar{c},(x, \lambda(x))$ é um par KKT associado a (PDV).

Demonstração. Suponha por absurdo que a tese é falsa. Então existe seqüência $c_{k} \rightarrow+\infty$ e uma seqüência limitada $\left\{x^{k}\right\} \subset \mathbb{R}^{n}$ tal que $0=W_{c_{k}}\left(x^{k}\right)$, e tal que $\left(x^{k}, \lambda\left(x^{k}\right)\right)$ não é solução KKT. Sem perda de generalidade, trocamos $\left\{x^{k}\right\}$ por uma subseqüência dela que converge para um $\bar{x}$. Mas pelo teorema 3.6 isso não é possível, pois ele garantiria que para um $K$ finito, $\left(x^{K}, \lambda\left(x^{K}\right)\right)$ é par KKT. 


\subsection{Um Caso Especial: Complementaridade Não-Linear}

Nesta seção vamos obter resultados mais precisos com a penalidade exata proposta, escrevendo explicitamente sua fórmula para o problema de complementaridade não-linear:

$$
F(x) \geq 0, \quad x \geq 0, \quad F(x)^{T} x=0
$$

Vamos primeiramente provar que (NCP) é uma Desigualdade Variacional em que $\mathcal{F}=\mathbb{R}_{+}^{n}$. Temos, para qualquer solução $\bar{x}$ de (NCP), que

$$
F(\bar{x})^{T}(y-\bar{x})=F(\bar{x})^{T} y-F(\bar{x})^{T} \bar{x}=F(\bar{x})^{T} y
$$

Se $y \geq 0$, como $F(\bar{x}) \geq 0, F(\bar{x})^{T} y \geq 0$. Então,

$$
F(\bar{x})^{T}(y-\bar{x}) \geq 0, \forall y \in \mathbb{R}_{+}^{n}
$$

Ou seja, $\bar{x}$ resolve a desigualdade variacional definida a partir de $F$ e com conjunto viável $\mathbb{R}_{+}^{n}$.

Por outro lado se $\bar{x}$ resolve o problema de desigualdade variacional

$$
x \in \mathbb{R}_{+}^{n} \text { t.q. } F(x)^{T}(y-x) \geq 0, \forall y \in \mathbb{R}_{+}^{n},
$$

temos que $\bar{x} \geq 0$ e tomando $y=0$ vem de $F(\bar{x})^{T} y \geq F(\bar{x})^{T} \bar{x}$ que

$$
\alpha \stackrel{\text { def }}{=} F(\bar{x})^{T} \bar{x} \leq 0
$$

Se existisse $i$ tal que $F_{i}(\bar{x})<0$, poderíamos tomar $y$ com a $i$-ésima coordenada de valor $\frac{\alpha-1}{F_{i}(\bar{x})}$, que é positivo, e as outras nulas e teríamos $y \in \mathbb{R}_{+}^{n}$ e $F(\bar{x})^{T} y=\alpha-1<\alpha=F(\bar{x})^{T} \bar{x}$, absurdo. Portanto $F(\bar{x}) \geq 0$ e $F(\bar{x})^{T} \bar{x} \geq 0$, o que nos dá, em conjunto com $(3.15), F(\bar{x})^{T} \bar{x}=0$, a condição de complementaridade. Lembrando que $\bar{x} \geq 0$, acabamos de mostrar que de fato (NCP) é equivalente a (3.14).

É essa última formulação que usaremos para resolver o problema, aplicando nossos resultados anteriores. Em particular temos que $g(x)=-x$ e a LICQ vale em todo domínio. Escrevendo explicitamente cada termo para cálculo da estimativa do multiplicador, obtemos: 


$$
\begin{gathered}
g(x)=-x \Longrightarrow \nabla g(x)=-I \\
N(x)=\left[\begin{array}{ll}
\nabla g(x) & \gamma G(x)
\end{array}\right]\left[\begin{array}{c}
\nabla g(x) \\
\gamma G(x)
\end{array}\right]=I+\gamma^{2} X^{2} \\
\lambda_{i}(x)=\left(-N^{-1}(x) \nabla g(x) F(x)\right)_{i}=\frac{F(x)_{i}}{1+\gamma^{2} x_{i}^{2}}
\end{gathered}
$$

Substituindo na fórmula da penalidade $W_{c}$, temos:

$$
\begin{array}{cc}
W_{c}(x)=F(x)+\nabla g(x)^{T} \lambda(x)+c \nabla g(x)^{T} \max \{g(x),-\lambda(x) / c\} & = \\
F(x)-\lambda(x)-\max \{-c x,-\lambda(x)\} & = \\
F(x)-\lambda(x)+\min \{c x, \lambda(x)\} & = \\
\min \{F(x)-\lambda(x)+c x, F(x)\} . &
\end{array}
$$

E colocando $F$ em evidência no primeiro termo, chegamos a

$$
W_{c}(x)_{i}=\min \left\{\frac{\gamma^{2} x_{i}^{2}}{1+\gamma^{2} x_{i}^{2}} F(x)_{i}+c x_{i}, F(x)_{i}\right\}, \quad i=1, \ldots, n .
$$

No caso de complementaridade, $W_{c}$ se reduziu a uma forma mais simples, um mínimo coordenada a coordenada entre $F$ e uma regularização sua com termo linear em $x$. Essa forma simplificada nos permite formular uma hipótese razoável que garante a limitação do conjunto de zeros de $W_{c}(\cdot)$ para $c$ grande o suficiente, o que tornará possível usar o último resultado da seção anterior.

Teorema 3.8. Suponha que vale uma das seguintes propriedades:

1. Existem $\rho>0$ e $M$ tais que $F(x)^{T} x \geq M$ para todo $x$ com $\|x\|>\rho$.

2. F é monótono e (NCP) tem solução.

Então existe $\bar{c}>0$ tal que $W_{c}(\cdot)$ é exato para todo $c>\bar{c}$, ou seja, suas soluções são soluções do $(\mathrm{NCP})$.

Demonstração. Suponha por absurdo que o resultado é falso. Então existe uma seqüência $c_{k} \rightarrow+\infty$, e uma seqüência $\left\{x^{k}\right\}$ tais que $W_{c_{k}}\left(x^{k}\right)=0$ e $x^{k}$ não é uma solução de (NCP). O teorema 3.6 garante que $\left\|x^{k}\right\| \rightarrow+\infty$. E a proposição 3.5 , mostra que $x^{k}$ nunca é viável.

Para cada $x^{k}$ podemos analisar $W_{c_{k}}\left(x^{k}\right)$ coordenada a coordenada, seguindo a expressão (3.16) e lembrando que todas elas devem se anular. Há três possibilidades:

1. Se $x_{i}^{k}>0$, então $F\left(x^{k}\right)_{i}=0$. 
Note que (3.16) implica $F\left(x^{k}\right)_{i} \geq W_{c_{k}}\left(x^{k}\right)_{i}=0$. Se $F\left(x^{k}\right)_{i}>0, W_{c_{k}}\left(x^{k}\right)_{i}$ seria o mínimo de dois números estritamente positivos, o que contradiz o fato de ele ser nulo, portanto $F\left(x^{k}\right)_{i}=0$.

2. Se $x_{i}^{k}=0$, então $F\left(x^{k}\right)_{i} \geq 0$.

Segue diretamente de $W_{c_{k}}\left(x^{k}\right)_{i}=0$ que $\min \left\{0, F\left(x^{k}\right)_{i}\right\}=0$. Isso é equivalente a $F\left(x^{k}\right)_{i} \geq 0$.

3. Se $x_{i}^{k}<0$, então $F\left(x^{k}\right)_{i}=-c_{k} \frac{1+\gamma^{2}\left(x_{i}^{k}\right)^{2}}{\gamma^{2} x_{i}^{k}}$.

Em primeiro lugar, se tivéssemos $F\left(x^{k}\right)_{i} \leq 0$, o primeiro termo do mínimo seria estritamente negativo e $W_{c_{k}}\left(x^{k}\right)_{i}$ seria o mínimo entre um número negativo e outro estritamente negativo, o que contradiria o fato de $W_{c_{k}}\left(x^{k}\right)_{i}=0$. Portanto $F\left(x^{k}\right)_{i}>0$ e o mínimo que define $W_{c_{k}}\left(x^{k}\right)_{i}=0$ tem de ser atingido no primeiro termo, o que nos leva a

$$
0=\frac{\gamma^{2}\left(x_{i}^{k}\right)^{2}}{1+\gamma^{2}\left(x_{i}^{k}\right)^{2}} F\left(x^{k}\right)_{i}+c_{k} x_{i}^{k}
$$

que nos dá a fórmula acima.

Uma consequência de só termos essas três possibilidades é que sempre $F\left(x^{k}\right) \geq 0$.

Consideremos agora que existem $\rho>0$ e $M$ tais que $F(x)^{T} x \geq M$ para $\|x\|>\rho$. As possibilidades acima nos mostram que nos dois primeiros casos ou $x_{i}^{k}$ ou $F\left(x^{k}\right)_{i}$ se anulam. Usando a fórmula de $F$ que aparece no terceiro caso, nós acabamos de provar que

$$
\begin{aligned}
& F\left(x^{k}\right)^{T} x^{k}=\sum_{i} x_{i}^{k} F\left(x^{k}\right)_{i}=\sum_{x_{i}^{k}<0}-c_{k} \frac{1+\gamma^{2}\left(x_{i}^{k}\right)^{2}}{\gamma^{2}} \\
& \rightarrow-\infty, \\
& \text { [lembrando que } x^{k} \text { não é viável] }
\end{aligned}
$$

e chegamos a uma contradição com nossa hipótese da limitação por $M$.

Para terminar, consideremos o caso em que $F$ é monótona e em que (NCP) tem uma 
solução $\bar{x}$. Temos

$$
\begin{aligned}
0 & \leq\left(F\left(x^{k}\right)-F(\bar{x})\right)^{T}\left(x^{k}-\bar{x}\right) & & \\
& =F\left(x^{k}\right)^{T} x^{k}-F\left(x^{k}\right)^{T} \bar{x}-F(\bar{x})^{T} x^{k}+F(\bar{x})^{T} \bar{x} & & \\
& \leq F\left(x^{k}\right)^{T} x^{k}-F(\bar{x})^{T} x^{k} & & {\left[F\left(x^{k}\right) \geq 0, \bar{x} \geq 0 \text { e } F(\bar{x})^{T} \bar{x}=0\right] } \\
& =\sum_{x_{i}^{k}<0}\left[-c_{k} \frac{1+\gamma^{2}\left(x_{i}^{k}\right)^{2}}{\gamma^{2}}\right]-\sum_{i=1}^{n} F(\bar{x})_{i} x_{i}^{k} & & {[\text { Eq. }(3.17)] } \\
& \leq \sum_{x_{i}^{k}<0}\left[-c_{k} \frac{1+\gamma^{2}\left(x_{i}^{k}\right)^{2}}{\gamma^{2}}-F(\bar{x})_{i} x_{i}^{k}\right] & & \\
& =\sum_{x_{i}^{k}<0} \frac{-c_{k}-\gamma^{2} x_{i}^{k}\left(c_{k} x_{i}^{k}+F(\bar{x})_{i}\right)}{\gamma^{2}} & &
\end{aligned}
$$

Suponha que existe um $x^{k}$ tal que para todo índice $i \operatorname{com} x_{i}^{k}<0$ temos $c_{k} x_{i}^{k}+F(\bar{x})_{i} \leq 0$, e portanto

$$
x_{i}^{k}<0 \Longrightarrow-\gamma^{2} x_{i}^{k}\left(c_{k} x_{i}^{k}+F(\bar{x})_{i}\right) \leq 0 \text {. }
$$

Nesse caso, a última soma acima seria estritamente menor do que 0, e chegamos a uma contradição.

Lembrando que $x_{k}$ é sempre inviável, sempre possui alguma coordenada negativa, e do absurdo acima vem que, para pelo menos uma dessas coordenadas $i$ com $x_{i}^{k}<0$, vale que $c_{k} x_{i}^{k}+F(\bar{x})_{i}>0$ e podemos escrever

$$
\begin{array}{rlrl}
0 & \leq \sum_{x_{i}^{k}<0} \frac{-c_{k}-\gamma^{2} x_{i}^{k}\left(c_{k} x_{i}^{k}+F(\bar{x})_{i}\right)}{\gamma^{2}} & \\
& \leq \sum_{x_{i}^{k}<0, c_{k} x_{i}^{k}+F(\bar{x})_{i}>0} \frac{-c_{k}-\gamma^{2} x_{i}^{k}\left(c_{k} x_{i}^{k}+F(\bar{x})_{i}\right)}{\gamma^{2}} & \\
& \leq \sum_{x_{i}^{k}<0, c_{k} x_{i}^{k}+F(\bar{x})_{i}>0} \frac{-c_{k}-\gamma^{2} x_{i}^{k} F(\bar{x})_{i}}{\gamma^{2}} & {\left[-c_{k}\left(x_{i}^{k}\right)^{2}<0\right]} \\
& \leq \sum_{x_{i}^{k}<0, c_{k} x_{i}^{k}+F(\bar{x})_{i}>0} \frac{-c_{k}+\gamma^{2} F(\bar{x})_{i}^{2} / c_{k}}{\gamma^{2}} & & {\left[c_{k} x_{i}^{k}+F(\bar{x})_{i}>0\right]} \\
& \rightarrow-\infty, &
\end{array}
$$

uma clara contradição. 
Garantimos em ambos os casos que uma seqüência $\left\{x^{k}\right\}$ que não contém solução não existe.

A hipótese de coercividade, a primeira do teorema acima, pedindo que $x^{T} F(x)$ seja limitado inferiormente fora de uma bola, não é tão artificial e restritiva quanto pode parecer de início. Uma das condições necessárias mais comuns para existência de soluções de equações generalizadas, como é o caso das desigualdades variacionais, pede que $x^{T} F(x) \geq 0$ para $x$ fora de uma bola, ou seja, uma condição mais forte do que a nossa [42].

Em particular, nossa condição vale para funções fracamente coercivas:

Proposição 3.9. Se $0 \in \operatorname{dom} F$ e $F$ é fracamente coerciva, ou seja, para todo $y \in \operatorname{dom} F$,

$$
\liminf _{\|x\| \rightarrow \infty} \frac{F(x)^{T}(x-y)}{\|x\|}>0
$$

então $\exists \rho>0$ tal que $F(x)^{T} x \geq 0$ por $\|x\|>\rho$, isto é, a hipótese 1 do teorema 3.8 é válida com $M=0$.

Demonstração. Como $0 \in \operatorname{dom} F$,

$$
\liminf _{\|x\| \rightarrow \infty} \frac{F(x)^{T} x}{\|x\|}>0
$$

e então $\exists \epsilon>0$ tal que

$$
\liminf _{\|x\| \rightarrow \infty} \frac{F(x)^{T} x}{\|x\|} \geq 2 \epsilon
$$

Pela definição do liminf, isso implica que existe $\rho>0$ tal que se $\|x\| \geq \rho, \frac{F(x)^{T} x}{\|x\|} \geq \epsilon$. Essa última inequação claramente implica que $F(x)^{T} x$ é positivo para $\|x\| \geq \rho$, e posso tomar $M=0$ na hipótese 1 do teorema 3.8 . 


\section{Capítulo 4}

\section{Conclusão}

Nessa dissertação, apresentamos uma revisão de conceitos de penalidades exatas para otimização e sua extensão para desigualdades variacionais. Em particular, mostramos como acoplar a estimativa de multiplicadores sugeridas por Glad e Polak [27] ao lagrangiano aumentado clássico para desigualdades variacionais sugerido por Auslender e Teboulle [2]. Obtivemos assim uma penalidade exata para (PDV). Os resultados mais finos de exatidão foram obtidos no caso de problemas de complementaridade não-linear, em que provamos que a penalidade é exata sempre que a função $F$ for monótona ou tiver propriedades razoáveis de coercividade.

Vale destacar que a penalidade exata sugerida não é obtida a partir da simples substituição do gradiente da função objetivo por uma função $F$ no gradiente da penalidade de otimização $w_{c}$. Com isso, evitamos que a fórmula da penalidade dependa do jacobiano da estimativa do multiplicador. Isso tem a conseqüência importante de criar uma fórmula que não envolve as hessianas das restrições nem o jacobiano de $F$. Esse fato pode ser relevante em implementações do método proposto, uma vez que as equações que devem ser resolvidas não envolvem termos de segunda ordem. Mesmo no caso de programação não-linear, ou seja, ao aplicarmos a penalidade exata desenvolvida em nosso trabalho para a resolução de problemas de otimização, os problemas a serem resolvidos são intrinsicamente mais simples do que aqueles sugeridos pela penalidade de Di Pillo e Grippo [15].

Esses primeiros resultados abrem então várias frentes naturais de pesquisa, entre as quais destacamos:

1. A busca por resultados de exatidão mais finos, como aqueles obtidos para o caso de complementaridade não-linear, para restrições baseadas em desigualdades convexas gerais. Por exemplo, quais as hipóteses de coercividade que devem ser satisfeitas para garantir 
que o conjunto de zeros de $W_{c}(x)$ seja limitado para $c$ suficientemente grande? Acreditamos que esse resultado é válido se o conjunto de soluções da desigualdade variacional for limitado. Mas, infelizmente, não conseguimos ainda demonstrar tal fato.

2. Analisar a possibilidade de se obter penalidades exatas baseadas em outros lagrangianos aumentados, como o lagrangiano aumentado cúbico sugerido por Eckstein e Ferris [18].

3. Seria muito importante procurar boas implementações de algoritmos para a resolução de desigualdades variacionais baseados em nossa penalidade. Para isso será necessário adaptar as estratégias de atualização do parâmetro $c$ desenvolvidas por Mukai, Glad e Polak [31, 27]. Note que aqui será também fundamental explorar a semi-suavidade da penalidade proposta, para que seja possível a aplicação de métodos de Newton generalizados. Aspectos de regularidade da penalidade também terão que ser estudados. O início desse estudo é feito no apêndice a seguir.

4. Por fim, como sugerido acima, seria interessante analisar o caso particular de programação não-linear, para ver se, de fato, a penalidade sugerida pode substituir as penalidades exatas de Di Pillo e Grippo na implementação de métodos numéricos. 


\section{Apêndice A}

\section{Métodos Numéricos}

Os resultados que apresentamos nesse apêndice foram desenvolvidos posteriormente à escrita inicial da dissertação e foram incorporados à versão final por sugestão da banca examinadora. O formato é menos didático do que os outros capítulos da dissertação e assemelha-se mais à fluência de um artigo.

\section{A.1 Reformulações NCP}

Vamos retomar a nossa penalidade exata para o problema de complementaridade:

$$
W_{c}(x)_{i}=\min \left\{\frac{\gamma^{2} x_{i}^{2}}{1+\gamma^{2} x_{i}^{2}} F(x)_{i}+c x_{i}, F(x)_{i}\right\}, \quad i=1, \ldots, n
$$

Sabemos, dos resultados do final da seção 3.3, que com algumas hipóteses sobre $F$ e $c$ podemos obter uma solução do problema de complementaridade não linear resolvendo a equação

$$
0=W_{c}(x)
$$

Observando a fórmula da penalidade, vemos que ela se baseia na função mínimo. Já, se resolvermos o problema

$$
0=\min \{F(x), x\},
$$

encontraremos um ponto que satisfaz $F(x) \geq 0, x \geq 0$ e para todo coordenada $i$ pelo menos um entre $F_{i}(x)$ e $x_{i}$ se anula. Ou seja, os zeros de $\mathcal{M}(x) \stackrel{\text { def }}{=} \min \{F(x), x\}$ também são zeros do problema de complementaridade. 
Poderíamos resolver o (NCP) resolvendo (A.3) ao invés do (A.2), que parece mais simples. Que vantagem pode haver em usar $W_{c}$ ?

Como $W_{c}$ foi construída a partir da escolha de um multiplicador de Lagrange que resolve, da melhor maneira possível, as condições KKT do problema de complementaridade, o cálculo de $W_{c}$ incorpora essa informação dual, o que pode gerar uma solução mais rapidamente. Verificaremos na seção A.3 o quanto essa estratégia é realmente útil.

Funções como $\mathcal{M}(x)$ ou $W_{c}(x)$, que quando zeradas encontram soluções do problema de complementaridade, são chamadas de funções NCP. Outra função NCP muito importante é a função de Fischer-Burmeister [22]

$$
\mathcal{F}(x) \stackrel{\text { def }}{=} F(x)+x+\sqrt{F^{2}(x)+x^{2}} .
$$

Uma das qualidades de $\mathcal{F}(x)$ é que ela é não-diferenciável apenas na origem, ao contrário de $\mathcal{M}(x)$, que é não-diferenciável em todo o conjunto em que $F(x)=x$.

Apesar de todas as reformulações NCP conhecidas serem não-diferenciáveis em pelo menos um ponto, muitas delas apresentam suficiente regularidade para serem semi-suaves, ou seja, possuem generalizações do jacobiano que recuperam informações de primeira ordem.

Dada uma função $F: \mathbb{R}^{n} \rightarrow \mathbb{R}^{m}$ localmente lipschitziana, a generalização de Clarke do jacobiano de $F$ no ponto $\bar{x}$, denotado por $\partial F(x)$, é o casco convexo de todos os limites dos jacobianos de pontos diferenciáveis convergindo para $x$ :

$$
\partial F(\bar{x})=\operatorname{co}\left\{\begin{array}{cc} 
& \\
\lim _{x \rightarrow \bar{x}} & \nabla F(x) \\
F \text { diferenciável em } x &
\end{array}\right\} .
$$

Uma função é chamada de semi-suave no ponto $\bar{x}$ quando é localmente lipschitziana em $\bar{x}$ e

$$
\begin{gathered}
\lim _{V \in} \partial F(\bar{x}+t h) \\
\quad h \rightarrow \bar{h} \\
\quad t \downarrow 0
\end{gathered}
$$


existe para todo $\bar{h} \in \mathbb{R}^{n}$. Essa condição é equivalente a

$$
\exists \rho \text { t.q. se }\|h\|<\rho, \forall V \in \partial F(x+h) \text {, vale } V h=F^{\prime}(x ; h)+o(\|h\|) \text {, }
$$

em que $F^{\prime}(x ; h)$ é a derivada direcional de $F$ calculada em $x$ na direção $h$ [38].

A função de Fischer-Burmeister é semi-suave, assim como $\mathcal{M}(x)$. Como a semi-suavidade é preservada por composição, $W_{c}(x)$ herda a semi-suavidade da mínimo. Essa semi-suavidade irá garantir a convergência de métodos de Newton adaptados, como veremos a seguir.

\section{A.2 Métodos de Resolução}

O método de Newton

$$
x^{k+1}=x^{k}-\left[\nabla T\left(x^{k}\right)\right]^{-1} T\left(x^{k}\right),
$$

é um método clássico para resolver a equação não-linear

$$
T(x)=0,
$$

em que $T: \mathbb{R}^{n} \rightarrow \mathbb{R}^{n}$ é uma função continuamente diferenciável. A direção $-\left[\nabla T\left(x^{k}\right)\right]^{-1} T\left(x^{k}\right)$ é chamada de direção de Newton e aponta diretamente para a solução em problema lineares.

Qi e Sun obtiveram resultados de convergência local do método de Newton em que $\nabla T\left(x^{k}\right)$ é substituído por um jacobiano generalizado $V_{k} \in \partial T\left(x^{k}\right)$, contanto que a função $T(x)$ seja semi-suave e possam sempre ser escolhidos $V_{k}$ inversíveis numa vizinhança da solução.

Poderíamos então utilizar esse método de Newton modificado para construir um algoritmo para resolver o problema NCP, $\operatorname{com} T(x)$ sendo $\mathcal{F}(x), \mathcal{M}(x)$ ou $W_{c}(x)$ [38]. Uma boa referência para a aplicação de variantes do método de Newton para funções semi-suaves na resolução de problemas de complementaridade não-linear é [13].

Por sua vez, Kanzow e Petra [29] desenvolveram uma nova função NCP baseada na função de Fischer-Burmeister mas com um termo extra que tenta forçar complementaridade. Denotaremos a função sugerida por Kanzow e Petra por $\mathcal{F}_{K P}$. Eles também propõem o uso de uma variante conhecida do método de Newton, chamado de Levenberg-Marquardt (LM), para a resolução da respectiva reformulação. Esse método, quando aplicado a problemas de complementaridade, obteve resultados ainda mais robustos do que aqueles baseados na função de Fischer-Burmeister pura. Para a sua globalização, ou seja para que ele convirja 
independentemente do ponto inicial, Kanzow e Petra usaram a função de mérito natural

$$
\frac{1}{2}\|T(x)\|^{2}
$$

Pela regra da cadeia, essa função será diferenciável quando o único ponto de não-diferenciabilidade de $T$ for a origem. Esse é o caso da função $\mathcal{F}(x)$ de Fischer-Burmeister, de $\mathcal{F}_{K P}(x)$, mas não é o caso da $\mathcal{M}(x)$ ou de $W_{c}(x)$.

Apesar disso, podemos adaptar o método de Newton, ou sua variante Levenberg-Marquardt, para que ele use uma função de mérito baseada em $\mathcal{F}$ ou $\mathcal{F}_{K P}$ porém empregando $\mathcal{M}(x)$ ou $W_{c}(x)$ para cômputo da direção de busca:

1. Calcula-se a direção de Newton (ou LM) para a função $\mathcal{M}(x)$ ou $W_{c}(x)$;

2. Calcula-se o ângulo entre essa direção e menos o gradiente da função de mérito $\frac{1}{2}\left\|\mathcal{F}_{K P}(x)\right\|^{2}$;

3. Se o ângulo for suficientemente pequeno, a direção calculada é uma boa direção de descida para a função de mérito. Faz-se a busca nessa direção. Se não, a busca é feita na direção de menos o gradiente da função de mérito.

Esse método permite que as direções sejam calculadas pela função de interesse, mas seu progresso é medido pela função de mérito que sabemos garantir a convergência.

\section{A.3 Resultados numéricos}

Com base na idéia de algoritmo construído na seção anterior, implementamos um método para comparar a penalidade exata, a função mínimo e a reformulação de Kanzow e Petra. O nosso código foi baseado em no programa LMMCP que implementa as idéias de Kanzow e Petra.

Nosso enfoque esteve em determinar a viabilidade da penalidade como reformulação para problemas de complementaridade, verificando sua capacidade de resolver os problemas mesmo sem termos determinado valores ótimos para $c$ e $\gamma$. Usamos $c=5$ e $\gamma=0.225$ em todos os testes com a penalidade. Os testes foram feitos com os problemas de complementaridade da MCPLIB, mesmo sem que eles satisfaçam todas as hipóteses do teorema de convergência, uma prática comum na literatura $[11,19]$.

Apresentamos a seguir o perfil de performance, que apresenta na abscissa a quantidade de iterações do método e na ordenada a proporção de problemas resolvidos. 


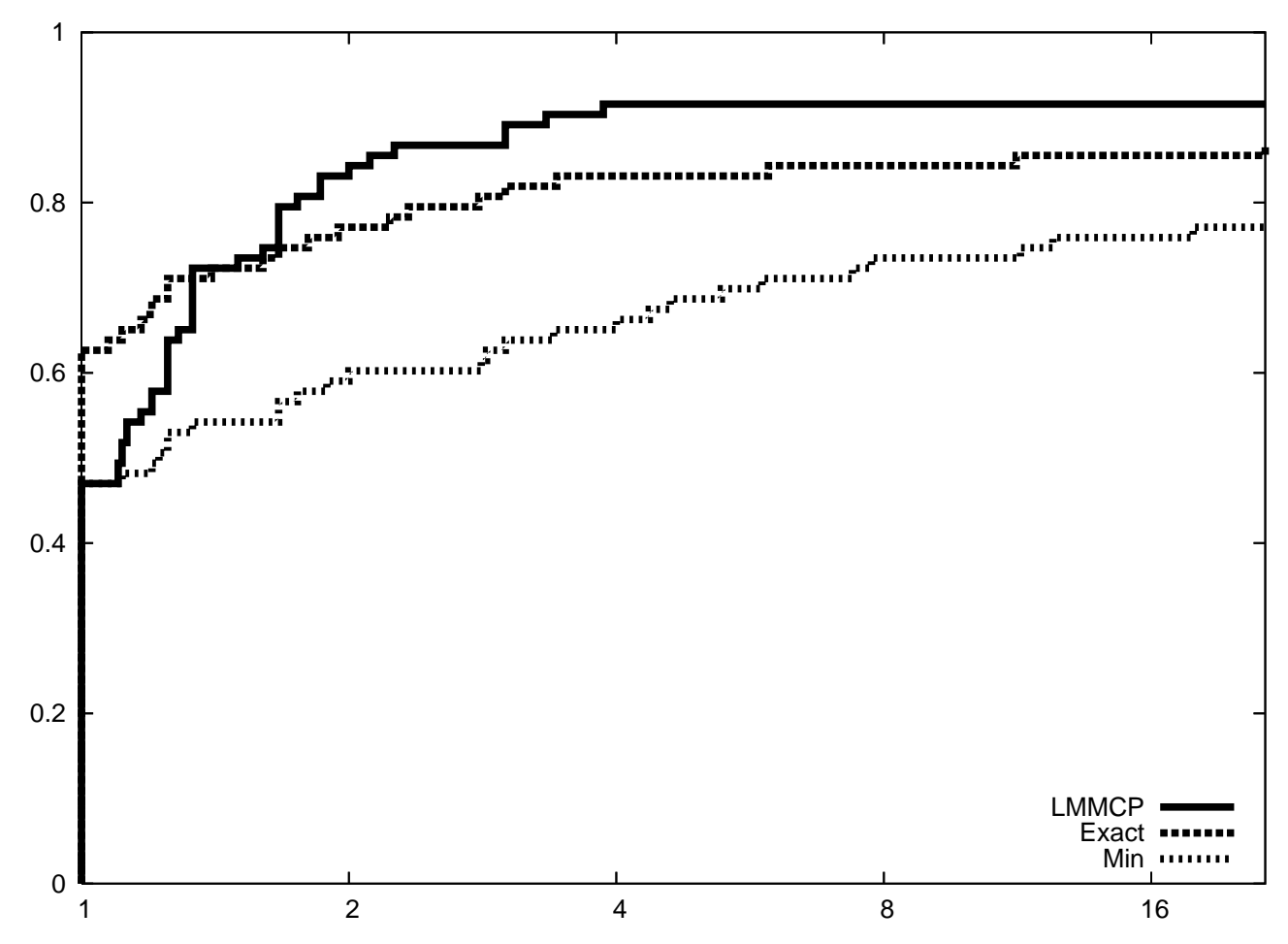

Nota-se que, como esperado, que o método LMMCP, baseado função de Fischer-Burmeister adaptada $\mathcal{F}_{K P}$, resolve muito mais problemas do que aqueles baseado na função mínimo, porém a função mínimo parece mais rápida nos problemas que é capaz de resolver. Já a variante que usa penalidade exata é a mais rápida de todas, resolvendo mais problemas com poucas iterações. Sua limitação está na robustez, pois resolve menos problemas do que o LMMCP. Observando os registros dos métodos, pudemos notar que muitas vezes estava-se usando a direção de Cauchy da função de mérito para se fazer a busca nas variantes que usam $\mathcal{M}(x)$ ou $W_{c}(x)$, o que indica que a respectiva direção de Newton muitas vezes não é uma boa direção de descida quando vista sob a ótica da função de mérito $\mathcal{F}_{K P}$.

\section{A.3.1 Melhorando a Robustez}

Para melhorar a robustez do método quando aplicado com a penalidade exata ou com a função mínimo, precisamos de alguma forma incorporar a qualidade da escolha da direção de Newton para $\mathcal{F}_{K P}$, mas sem perder a velocidade proporcionada pela informação dual e pela função 
mínimo. Além disso, não queremos calcular duas direções de Newton diferentes, resolvendo dois sistemas lineares por iteração. Na tentativa de prever quando a direção calculada a partir de $W_{c}$ ou $\mathcal{M}$ pode ser ruim, vamos comparar as direções dadas pelos gradientes da função de mérito, $\nabla\left(\frac{1}{2}\left\|\mathcal{F}_{K P}(x)\right\|^{2}\right)$, e a função mérito associada a reformulação, p. ex. $\nabla\left(\frac{1}{2}\left\|W_{c}(x)\right\|^{2}\right)$. Vamos usar o ângulo formado por esses vetores para decidir qual direção de Newton calcular.

1. Calcula-se o ângulo entre o gradiente da função de mérito $\frac{1}{2}\left\|\mathcal{F}_{K P}(x)\right\|^{2}$ e menos o gradiente da função de mérito associada a reformulação.

2. Se o ângulo for suficientemente pequeno, a função de reformulação é apropriada para cálculo da direção de descida, e calcula-se a direção de LM para a função de reformulação. Se não, calcula-se a direção LM para a Fischer-Burmeister adaptada.

3. Calcula-se o ângulo entre essa direção e menos o gradiente da função de mérito $\frac{1}{2}\left\|\mathcal{F}_{K P}(x)\right\|^{2}$.

4. Se o ângulo for suficientemente pequeno, essa é um boa direção de descida e faz-se a busca na direção calculada. Se não, a busca é feita na direção de menos o gradiente da função de mérito.

Como comprova o perfil de performance a seguir, de fato essa modificação permitiu que a robustez do método LMMCP fosse transmitida para a penalidade exata e para a função mínimo. Além disso, a boa velocidade da penalidade não foi alterada e o método é quase sempre mais rápido do que o LMMCP, resolvendo a mesma proporção de problemas. 


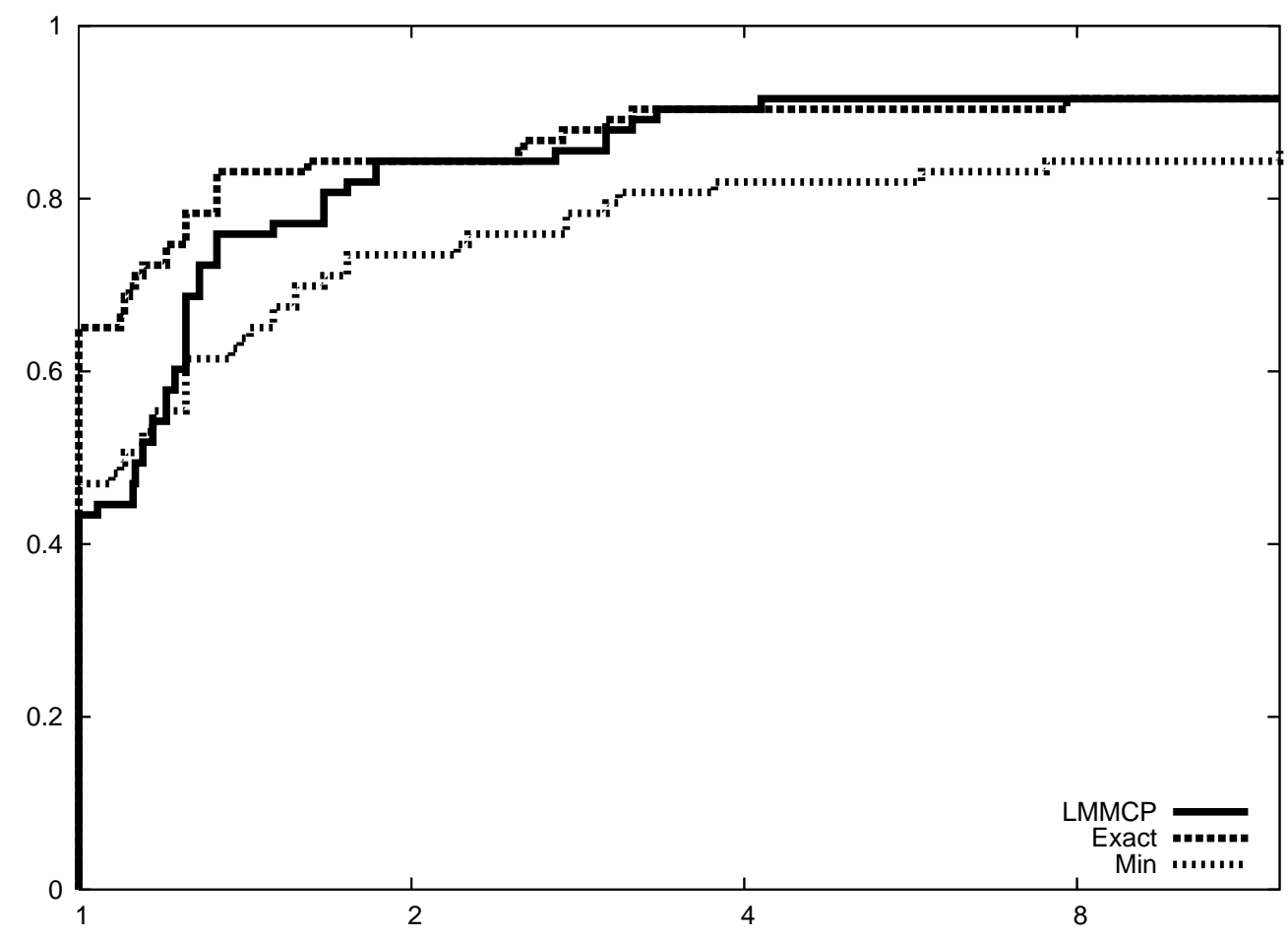




\section{Referências Bibliográficas}

[1] H. Attouch and M. Théra. A general duality principle for the sum of two operators. $J$. Convex Anal., 3(1):1-24, 1996.

[2] A Auslender, M. Teboulle, and S. Ben-Tiba. Modified lagrangian methods for variational inequality problems. pre-print.

[3] A. Auslender, M. Teboulle, and S. Ben-Tiba. A logarithmic-quadratic proximal method for variational inequalities. Computational Optimization and Applications, 12:31-40, 1999.

[4] Alfred Auslender and Marc Teboulle. Lagrangian duality and related multiplier methods for variational inequality problems. SIAM J. Optim., 10(4):1097-1115, 2000.

[5] Alfred Auslender and Marc Teboulle. Asymptotic cones and functions in optimization and variational inequalities. Springer Monographs in Mathematics. Springer-Verlag, New York, 2003.

[6] Alfred Auslender, Marc Teboulle, and Sami Ben-Tiba. Interior proximal and multiplier methods based on second order homogeneous kernels. Math. Oper. Res., 24(3):645-668, 1999.

[7] Mokhtar S. Bazaraa, Hanif D. Sherali, and C. M. Shetty. Nonlinear programming. WileyInterscience [John Wiley \& Sons], Hoboken, NJ, third edition, 2006. Theory and algorithms.

[8] D. Bertsekas. Constrained Optimization and Lagrange Multipliers. Academic Press, New York, 1982.

[9] D. Bertsekas. Nonlinear Programming. Athena Scientific, 1995. 
[10] D. Bertsekas and A. Ozdaglar-Koksal. Enhanced optimality conditions and exact penalty functions, 2000 .

[11] Stephen C. Billups, Steven P. Dirkse, and Michael C. Ferris. A comparison of large scale mixed complementarity problem solvers. Comput. Optim. Appl., 7(1):3-25, 1997. Computational issues in high performance software for nonlinear optimization (Capri, 1995).

[12] Stephen Boyd and Lieven Vandenberghe. Convex optimization. Cambridge University Press, Cambridge, 2004.

[13] T. De Luca, F. Facchinei, and Kanzow C. A theoretical and numerical comparison of some semismooth algorithms for complementarity problems. Computational Optimization and Applications, 16:173-205, 2000.

[14] G. di Pillo and L. Grippo. A new class of augmented Lagrangians in nonlinear programming. SIAM J. Control Optim., 17(5):618-628, 1979.

[15] G. Di Pillo and L. Grippo. A continuously differentiable exact penalty function for nonlinear programming problems with inequality constraints. SIAM J. Control Optim., 23(1):72-84, 1985.

[16] G. Di Pillo and L. Grippo. Exact penalty functions in constrained optimization. SIAM J. Control Optim., 27(6):1333-1360, 1989.

[17] G. Di Pillo, L. Grippo, and F. Lampariello. A method for solving equality constrained optimization problems by unconstrained minimization. In Optimization techniques (Proc. Ninth IFIP Conf., Warsaw, 1979), Part 2, volume 23 of Lecture Notes in Control and Information Sci., pages 96-105. Springer, Berlin, 1980.

[18] Jonathan Eckstein and Michael C. Ferris. Smooth methods of multipliers for complementarity problems. Math. Program., 86(1, Ser. A):65-90, 1999.

[19] Jonathan Eckstein and Michael C. Ferris. Smooth methods of multipliers for complementarity problems. Math. Program., 86(1, Ser. A):65-90, 1999.

[20] I. Ekeland. On the variational principle. J. Math. Anal. Appl., 47:324-353, 1974.

[21] Francisco Facchinei and Jong-Shi Pang. Finite-dimensional variational inequalities and complementarity problems, Vol. I. Springer Series in Operations Research. SpringerVerlag, New York, 2003. 
[22] A. Fischer. A special Newton-type optimization method. Optimization, 24(3-4):269-284, 1992.

[23] R. Fletcher. A class of methods for nonlinear programming with termination and convergence properties. In Integer and nonlinear programming, pages 157-175. North-Holland, Amsterdam, 1970.

[24] R. Fletcher. A class of methods for non-linear programming. III. Rates of convergence. In Numerical methods for non-linear optimization (Conf., Dundee, 1971), pages 371-381. Academic Press, London, 1972.

[25] R. Fletcher. An exact penalty function for nonlinear programming with inequalities. Math. Programming, 5:129-150, 1973.

[26] R. Fletcher and Shirley A. Lill. A class of methods for nonlinear programming. II. Computational experience. In Nonlinear Programming (Proc. Sympos., Univ. of Wisconsin, Madison, Wis., 1970), pages 67-92. Academic Press, New York, 1970.

[27] Torkel Glad and Elijah Polak. A multiplier method with automatic limitation of penalty growth. Math. Programming, 17(2):140-155, 1979.

[28] S. P. Han and O. L. Mangasarian. Exact penalty functions in nonlinear programming. Math. Programming, 17(3):251-269, 1979.

[29] Christian Kanzow and Stefania Petra. On a semismooth least squares formulation of complementarity problems with gap reduction. Optim. Methods Softw., 19(5):507-525, 2004.

[30] Umberto Mosco. Dual variational inequalities. J. Math. Anal. Appl., 40:202-206, 1972.

[31] H. Mukai and E. Polak. A quadratically convergent primal-dual algorithm with global convergence properties for solving optimization problems with equality constraints. Math. Programming, 9(3):336-349, 1975.

[32] H. Mukai and E. Polak. A quadratically convergent primal-dual algorithm with global convergence properties for solving optimization problems with equality constraints. Math. Programming, 9(3):336-349, 1975.

[33] Jorge Nocedal and Stephen J. Wright. Numerical optimization. Springer Series in Operations Research. Springer-Verlag, New York, 1999. 
[34] T. Pennanen. Dualization of Monotone Generalized Equations. PhD thesis, University of Washington, 1999.

[35] Teemu Pennanen. Dualization of generalized equations of maximal monotone type. SIAM J. Optim., 10(3):809-835, 2000.

[36] Tomasz Pietrzykowski. An exact potential method for constrained maxima. SIAM J. Numer. Anal., 6:299-304, 1969.

[37] Li Qun Qi. Convergence analysis of some algorithms for solving nonsmooth equations. Math. Oper. Res., 18(1):227-244, 1993.

[38] Li Qun Qi and Jie Sun. A nonsmooth version of Newton's method. Math. Programming, 58(3, Ser. A):353-367, 1993.

[39] R. T. Rockafellar. Convex Analysis. Princeton University Press, 1970.

[40] R. T. Rockafellar. Augmented lagrangians and applications of the proximal point algorithm in convex programming. Mathematics of Operations Research, 1:97-116, 1976.

[41] R. T Rockafellar. Monotone operators and the proximal point algorithm. SIAM Journal on Control and Optimization, 14:887-898, August 1976.

[42] R. Tyrrell Rockafellar and Roger J.-B. Wets. Variational analysis, volume 317 of Grundlehren der Mathematischen Wissenschaften [Fundamental Principles of Mathematical Sciences]. Springer-Verlag, Berlin, 1998.

[43] P. J. S. Silva. Método de ponto proximal e separadores. Master's thesis, Instituto de Matemática e Estatística, Universidade de São Paulo, 1997.

[44] P. J. S. Silva. Tópicos em Métodos de Ponto Proximal. PhD thesis, Instituto de Matemática e Estatística, Universidade de São Paulo, 2000.

[45] P. J. S. Silva and J. Eckstein. Double-regularization proximal methods, with complementarity applications. Computational Optimization and Applications, 33:115-156, 2006.

[46] W. I. Zangwill. Nonlinear Programming: An Unified Aproach. Prentice Hall, Englewood Cliffs, 1969.

[47] Willard I. Zangwill. Non-linear programming via penalty functions. Management Sci., 13:344-358, 1967. 